\title{
Stable and Entropy Satisfying Approximations for Transonic Flow Calculations
}

\author{
By Bjorn Engquist* and Stanley Osher**
}

\begin{abstract}
Finite difference approximations for the small disturbance equation of tran sonic flow are developed and analyzed. New schemes of the Cole-Murman type are presented for which nonlinear stability is proved. The Cole-Murman scheme may have entropy violating expansion shocks as solutions. In the new schemes the switch between the subsonic and supersonic domains is designed such that these nonphysical shocks are guaranteed not to occur. Results from numerical calculations are given which illustrate these conclusions.
\end{abstract}

1. Introduction. The small disturbance equation of transonic flow is a common model for describing subsonic and supersonic flow close to the local speed of sound (see [2]). The flow is assumed to be that of an inviscid perfect gas. The small disturbance equation is derived via an asymptotic expansion around constant flow (see e.g. [2]). The differential equations for the steady state case and for the low frequency time-dependent case can be written, respectively,

$$
\begin{gathered}
\left(K \Phi_{x}-1 / 2(\gamma+1) \Phi_{x}^{2}\right)_{x}+\Phi_{y y}=0 \\
\left(K \Phi_{x}-1 / 2(\gamma+1) \Phi_{x}^{2}\right)_{x}+\Phi_{y y}-2 \Phi_{t x}=0
\end{gathered}
$$

where $\Phi(x, y, t)$ is the velocity potential, $K$ and $\gamma$ are positive constants.

During the last few years many numerical calculations using these equations have been presented (see [1], [2], [6], [8], [12] and the bibliographies in these papers). The equations are used as models for more complex systems since they contain many important phenomena such as shock formation. Moreover, the steady state part is of mixed hyperbolic and elliptic type. The equations are also of direct practical importance and typical applications are: Flow around an airplane flying close to Mach number 1, flows in nozzles and over turbine blades and propellers.

In the numerical calculations the differential equations are replaced by difference approximations. The most common schemes are type dependent. That is, different formulas are used in the subsonic and the supersonic domains. In this way it has been possible to avoid most nonphysical shocks and to keep the shock front sharper than in the case of uniform schemes. Variants of the Cole-Murman difference scheme [12] have been the basis for many successful calculations (see [1] , [6] , [8]). However, in [6] it is pointed out that the Cole-Murman scheme does admit entropy violating

Received April 6, 1979.

AMS (MOS) subject classifications (1970). Primary 65 M10; Secondary 76H05, 65 M05.

* Partially supported by the Swedish Natural Science Research Council (NFR-2711-018).

* Partially supported by NSF Grant MCS-78-01252. 
shocks as solutions, and furthermore in [1] and [8] instabilities are reported even if the schemes should be stable according to linear stability analysis. It is the purpose of this paper to give simple modifications of the Cole-Murman scheme which do not admit entropy violating shocks as solutions and to present rigorous stability analysis for the full nonlinear problem.

This type of analysis increases the understanding of the nonlinear numerical phenomena and is useful in the design of new schemes for the small disturbance equation or similar problems. Two new schemes of fractional step type in conservation form are presented.

For both of them the $x$-step is explicit and the $y$-step is implicit. In the implicit step a sequence of linear tridiagonal systems is solved. The nonlinear $x$-step is type dependent and upwind differencing is used away from the interface between supersonic and subsonic domains.

This step also keeps the shock profile sharp. A shock is spread over at most two grid points. In the first scheme the $x$-step is monotone and hence, as a separate algorithm, this difference approximation is convergent for Courant numbers up to one (see [3]).

Our version of the Cole-Murman scheme is as easy to program as the original and can be written in a particularly simple form. The $y$-differencing is unchanged and the $x$-differencing is changed only at sonic points or at supersonic-subsonic shock points. The fractional step algorithm presented below, although useful, is secondary in importance to the new $x$-differencing. Our time independent scheme is outlined in (2.8) below while that of Cole-Murman is described in (2.11) below.

In the following section the first difference scheme for the steady state equation (1.1) is presented. The algorithm is proved not to have entropy violating solutions.

A piecewise smooth entropy violating solution to (1.1) has an expansion shock. This is a jump discontinuity across which $\Phi_{x}$, the velocity in the $x$-direction increases, i.e., the inequality

$$
\Phi_{x x}<\infty
$$

is violated.

It is easy to show (see e.g. [11]), that in this piecewise smooth case, inequality (1.3) is equivalent to the following:

$$
\frac{\partial}{\partial x}\left(\frac{\Phi_{y}^{2}}{2}-K \frac{\Phi_{x}^{2}}{2}+(\gamma+1) \frac{\Phi_{x}^{3}}{3}\right)-\frac{\partial}{\partial y}\left(\Phi_{x} \Phi_{y}\right) \leqslant 0,
$$

which we take to be the entropy inequality, in view of its generality.

The entropy inequality for the time-dependent case (1.2) is taken to be

$$
\frac{\partial}{\partial t} \Phi_{x}^{2}+\frac{\partial}{\partial x}\left(\frac{\Phi_{y}^{2}}{2}-K \frac{\Phi_{x}^{2}}{2}+(\gamma+1) \frac{\Phi_{x}^{3}}{3}\right)-\frac{\partial}{\partial y}\left(\Phi_{x} \Phi_{y}\right) \leqslant 0 .
$$

See [11] for a discussion of the equivalence of various entropy conditions.

Section 3 deals with the time-dependent case (1.2). The algorithm can be used for time-dependent problems or as an iteration scheme for steady state problems. Each fractional step in the ADI-scheme is proved to be stable in $L^{2}$ with constant 1 . Thus 
the full nonlinear scheme is $L^{2}$-stable. Furthermore, the time-dependent entropy condition is satisfied by the limit solution. The analysis is based on energy estimates.

In Section 4 the second new version of the Cole-Murman scheme is presented together with the corresponding crucial estimates which were needed in the proofs of Sections 2 and 3.

The one-dimensional ( $y$-independent) model is studied in Section 5. Existence and uniqueness for the steady state difference approximation is proved. A numerical test with several time-dependent difference schemes is given. Among other observations we show that the original Cole-Murman scheme may converge in a stable way to entropy violating shocks and the Lax-Friedrichs scheme may have time oscillatory nonconverging solutions. The monotone scheme given in this paper converges rapidly to the exact analytic solution for all grid points but one on each side of the shock.

The last section contains numerical calculations of the two-dimensional problem. Results displaying the shock profile are presented together with a list of computational comments.

2. The Entropy Inequality for the Time-Independent Scheme. In this section we shall analyze a difference approximation to the steady two-dimensional small disturbance equation (1.1)

$$
\left(K \Phi_{x}-1 / 2(\gamma+1) \Phi_{x}^{2}\right)_{x}+\Phi_{y y}=0
$$

for $K, \gamma$ positive constants.

We shall first consider only local solutions of (2.1). Boundaries, or inversion of the system, and a time-dependent model, will be dealt with in the following section.

We let $u$ and $v$ be the velocities in the $x$ and $y$ direction, respectively, and

$$
\Phi_{x}=u, \quad \Phi_{y}=v .
$$

We define the convex function

$$
f(u)=-K u+(\gamma+1) \frac{u^{2}}{2} .
$$

The equation (2.1) can be rewritten

$$
-(f(u))_{x}+v_{y}=0 .
$$

This equation is hyperbolic if $u>\bar{u}$ and elliptic if $u<\bar{u}$, for $\bar{u}$ the sonic speed

$$
\bar{u}=\frac{K}{\gamma+1} .
$$

We shall solve a difference approximation for a discrete potential function $\varphi_{j k}$ approximating $\Phi(j \Delta x, k \Delta y)$. Here we have defined a grid $\left(x_{j}, y_{k}\right)=(j \Delta x, k \Delta y)$ for $j, k=0, \pm 1, \pm 2, \ldots$.

We also define discrete velocities

$$
D_{-}^{x} \varphi_{j k}=u_{j k}, \quad D_{-}^{y} \varphi_{j k}=v_{j k}
$$

Here and in what follows for any grid function $f_{j k}$ 


$$
D_{ \pm}^{x} f_{j k}=\mp\left(\frac{f_{j k}-f_{j \pm 1, k}}{\Delta x}\right), \quad D_{ \pm}^{x} f_{j k}=\mp\left(f_{j k}-f_{j \pm 1, k}\right) .
$$

$D_{ \pm}^{y}$ and $\Delta_{ \pm}^{y}$ are defined analogously.

We may now define our modification of the Cole-Murman difference approximation to (2.1).

$$
-D_{-}^{x} f\left(\max \left(u_{j k}, \bar{u}\right)\right)-D_{+}^{x} f\left(\min \left(u_{j k}, \bar{u}\right)\right)+D_{+}^{y} v_{j k}=0 .
$$

We may define the functions:

$$
\begin{aligned}
& \text { (a) } f_{1}(u)=f(u) \quad \text { if } u \geqslant \bar{u} \text {, } \\
& f_{1}(u)=f(\bar{u}) \quad \text { if } u \leqslant \bar{u}, \\
& f_{2}(u)=f(u) \quad \text { if } u \leqslant \bar{u}, \\
& f_{2}(u)=f(\bar{u}) \quad \text { if } u \geqslant \bar{u} \text {. }
\end{aligned}
$$

Thus, $f_{1}, f_{2}$ are $C^{1}$ and piecewise smooth functions with $f_{1}(u)+f_{2}(u) \equiv f(u)$ $+f(\bar{u})$; and we may rewrite (2.8).

It is easy to see that this scheme is consistent with (2.1), in conservation form, first order accurate in the hyperbolic (supersonic) region, and second order accurate in the elliptic (subsonic) region. Moreover, if $u_{j-1, k}, u_{j k}$, and $u_{j+1, k}$ all lie in the supersonic region the scheme agrees with that of Cole-Murman [12] and the difference stencil as described in Figure 2.1 is implicit hyperbolic. If these three values all lie in the subsonic region the scheme is again the same as the Cole-Murman scheme and the difference stencil is of standard elliptic type, again see Figure 2.1.

The significant differences between the two schemes occur at parabolic and shock points.

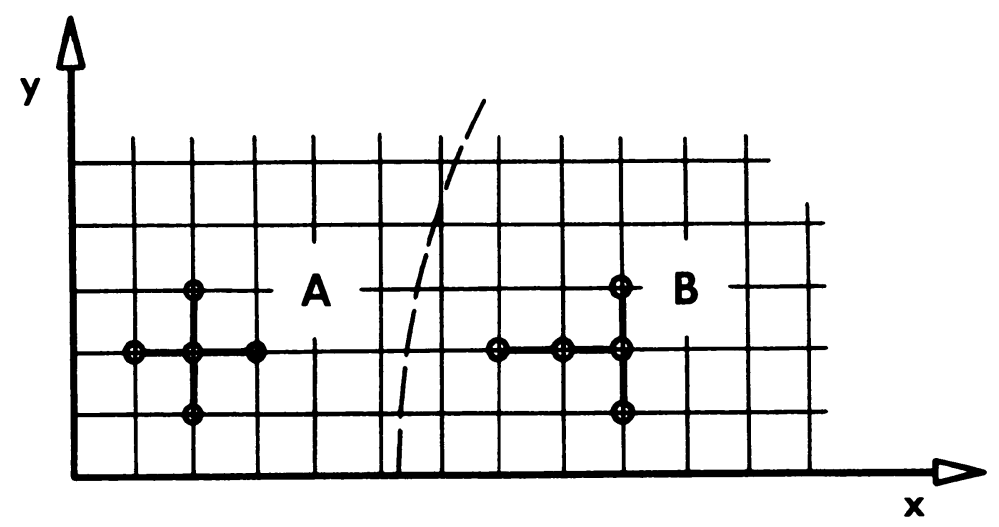

FIgURE 2.1

Part of the computational grid for $\varphi$ with a subsonic (A) and a supersonic (B) difference stencil. The dashes represent the sonic line. 
Recall, the Cole-Murman scheme in conservation form may be written

$$
-D_{+}^{x} f\left(u_{j k}\right)+\Delta_{-}^{x} \theta_{j k} D_{+}^{x} f\left(u_{j k}\right)+D_{+}^{y} v_{j k}=0,
$$

where

$$
\theta_{j k} \equiv 1 \text { if } \frac{u_{j k}+u_{j+1, k}}{2} \geqslant \bar{u}, \quad \theta_{j k} \equiv 0 \text { if } \frac{u_{j k}+u_{j+1, k}}{2}<\bar{u} .
$$

We call $\left(u_{j k}+u_{j+1, k}\right) / 2=u_{j+1 / 2, k}$ in what follows.

The following table gives us the $x$-differencing at shock and sonic points for our scheme E-O, and for the Cole-Murman scheme.

\begin{tabular}{lll}
\hline Values of $u$ & \multicolumn{1}{c}{ C-M Differencing } & E-O Differencing \\
\hline (1) $u_{j-1, k}, u_{j k}>\bar{u}>u_{j+1, k}$ & $\begin{array}{l}\text { if } u_{j+1 / 2, k}<\bar{u}:-2 D_{0}^{x} f\left(u_{j k}\right) \\
\text { if } u_{j+1 / 2, k} \geqslant \bar{u}:-D_{-}^{x} f\left(u_{j k}\right)\end{array}$ & $-D_{-}^{x} f\left(u_{j k}\right)-D_{+}^{x} f_{2}\left(u_{j k}\right)$ \\
\hline & if $u_{j-1 / 2, k}<\bar{u}:-D_{+}^{x} f\left(u_{j k}\right)$ & $-D_{-}^{x} f_{1}\left(u_{j k}\right)-D_{+}^{x} f\left(u_{j k}\right)$ \\
(2) $u_{j-1, k}>\bar{u}>u_{j k}, u_{j+1, k}$ & if $u_{j-1 / 2, k} \geqslant \bar{u}:-2 D_{0}^{x} f\left(u_{j k}\right)$ & \\
\hline & if $u_{j+1 / 2, k}<\bar{u}:-D_{+}^{x} f\left(u_{j k}\right)$ & $-D_{+}^{x} f_{2}\left(u_{j k}\right)$ \\
\hline & if $u_{j+1 / 2, k} \geqslant \bar{u}: 0$ & \\
\hline & if $u_{j-1 / 2}<\bar{u}: 0$ & $-D_{-}^{x} f_{1}\left(u_{j k}\right)$. \\
\hline
\end{tabular}

Cases (3) and (4) correspond to expansion shocks.

The E-O differencing will be shown to rule out such solutions in the limit as $\Delta x, \Delta y \rightarrow 0$.

This is the content of the main theorem of this section.

THEOREM 2.1. Suppose $\varphi_{j k}$ is determined by (2.8) and suppose $\varphi_{j k}, D_{-}^{x} \varphi_{j k}$, and $D_{-}^{y} \varphi_{j k}$ converge boundedly a.e. as $\Delta x, \Delta y \rightarrow 0$ to $\Phi, \Phi_{x}$, and $\Phi_{y}$, respectively.

Then $\Phi$ is a weak solution of (2.1) which satisfies the entropy inequality: for any $\rho \in C_{0}^{1}$ with $\rho \geqslant 0$

$$
\iint\left[\rho_{x} F\left(\Phi_{x}, \Phi_{y}\right)+\rho_{y} G\left(\Phi_{x}, \Phi_{y}\right) d x d y\right] \geqslant 0
$$

Here

$$
F\left(\Phi_{x}, \Phi_{y}\right)=\frac{\Phi_{y}^{2}}{2}-K \frac{\Phi_{x}^{2}}{2}+(\gamma+1) \frac{\Phi_{x}^{3}}{3}
$$

and $G\left(\Phi_{x}, \Phi_{y}\right)=-\Phi_{x} \Phi_{y}$.

The fact that $\Phi$ is a weak solution of (2.1) follows from a result of Lax and Wendroff [9]. We shall prove the entropy inequality from the following two lemmas.

Lemma 2.1. Let $\rho(x) \in C_{0}^{1}$ with $\rho \geqslant 0$ and let $f^{\prime \prime}(u)>0$ with $f^{\prime}(\bar{u})=0$. Then 


$$
\begin{aligned}
\sum_{j} \rho(j \Delta x) u_{j}\left[\Delta_{+} f\left(\min \left(u_{j}, \bar{u}\right)\right)+\Delta_{-} f\left(\max \left(u_{j}, \bar{u}\right)\right)\right] \\
=-\sum_{j}\left(\Delta_{-} \rho(j \Delta x)\right) \int_{0}^{u_{j}} s f^{\prime}(s) d s \\
\quad-\sum_{j}(\rho(j \Delta x)) \int_{u_{j}}^{u_{j+1}}\left[s-u_{j}-\left(\Delta_{+} u_{j}\right) \chi(s)\right] f^{\prime}(s) d s \\
\quad+\sum_{j}\left(\Delta_{+} \rho(j \Delta x)\right) u_{j+1} \int_{u_{j}}^{u_{j+1}} \chi(s) f^{\prime}(s) d s,
\end{aligned}
$$

where

$$
\chi(s) \equiv 1 \quad \text { if } s \geqslant \bar{u}, \quad \chi(s) \equiv 0 \text { if } s<\bar{u} .
$$

LEMMA 2.2. Let $\rho(x, y) \in C_{0}^{1}$ with $\rho \geqslant 0$. Then if $D_{-}^{\underline{y}} u=D_{-}^{x} v$ it follows that

$$
\begin{aligned}
\sum_{j, k} \rho(j \Delta x, k \Delta y) u_{j k}\left(\Delta_{+}^{y} v_{j k}\right) \\
=-\sum_{j, k}\left(\Delta_{-}^{y} \rho(j \Delta x, k \Delta y)\right) u_{j k} v_{j k} \\
+\frac{1}{2} \frac{\Delta y}{\Delta x} \sum_{j, k}\left(\Delta_{-}^{x} \rho(j \Delta x,(k-1) \Delta y)\right) v_{j-1, k}^{2} \\
\quad-\frac{1}{2} \frac{\Delta y}{\Delta x} \sum_{j, k} \rho(j \Delta x,(k-1) \Delta y)\left(\Delta_{-}^{x} v_{j k}\right)^{2} .
\end{aligned}
$$

We shall prove these lemmas below.

Proof of Theorem 2.1. We may now prove the entropy inequality. Let $\varphi_{j k}$ solve (2.8). Multiply (2.8) by $\rho(j \Delta x, k \Delta y) u_{j k} \Delta x \Delta y$ and sum. The two previous lemmas give us

$$
\begin{aligned}
& \sum\left(\Delta_{-}^{x} \frac{\rho(j \Delta x, k \Delta y)}{\Delta x}\right)\left[-K \frac{u_{j k}^{2}}{2}+\frac{(\gamma+1) u_{j k}^{3}}{3}+\frac{1}{2} v_{j-1, k+1}^{2}\right] \Delta x \Delta y \\
& +\sum\left(\frac{\Delta_{-}^{y} \rho(j \Delta x, k \Delta y)}{\Delta y}\right)\left[-u_{j k} v_{j k}\right] \Delta x \Delta y \\
& =\sum \rho(j \Delta x, k \Delta y)\left[\int_{u_{j, k}}^{u_{j+1, k}} \frac{\left[u_{j k}-s+\Delta_{+}^{x} u_{j k} \chi(s)\right]}{\Delta x} f^{\prime}(s) d s\right. \\
& \left.+\frac{1}{2}\left(\frac{\Delta_{-}^{x} u_{j k}}{\Delta x}\right)^{2}\right] \Delta x \Delta y \\
& +\sum \frac{\left(\Delta_{-}^{x} \rho(j \Delta x, k \Delta y)\right)}{\Delta x} u_{j k} \int_{u_{j-1, k}}^{u_{j k}} \chi(s) f^{\prime}(s) d s \Delta x \Delta y \\
& =[\mathrm{I}]+[\mathrm{II}] \text {. }
\end{aligned}
$$

By the Lebesgue dominated convergence theorem it is clear that

$$
\lim _{\Delta x, \Delta y \rightarrow 0}[\mathrm{II}]=0 .
$$


The theorem will follow if we can show that

$$
\varliminf_{\Delta x, \Delta y \rightarrow 0}[I] \geqslant 0 \text {. }
$$

It thus remains only to show that

$$
\int_{u_{j, k}}^{u_{j+1, k}}\left[u_{j k}-s+\Delta_{+} u_{j k} \chi(s)\right] f^{\prime}(s) d s \geqslant 0,
$$

which is true if for each $s$ in the interval $\left(u_{j k}, u_{j, k+1}\right)$

$$
\left(\left|\Delta_{+} u_{j k}\right| \chi(s)-\left|s-u_{j k}\right|\right) f^{\prime}(s) \geqslant 0
$$

or

$$
\left(\left|s-u_{j+1, k}\right| \chi(s)+\left|s-u_{j k}\right|(\chi(s)-1)\right) f^{\prime}(s) \geqslant 0 .
$$

This follows immediately from the definition of $\chi(s)$.

Proof of Lemma 2.1. We have

$$
\begin{aligned}
\sum \rho(j \Delta x) u_{j}\left[\Delta_{+} f\left(\min \left(u_{j}, \bar{u}\right)\right)+\Delta_{-} f\left(\max \left(u_{j}, \bar{u}\right)\right)\right] \\
=\sum \rho(j \Delta x) u_{j}\left[\Delta_{+} f\left(u_{j}\right)-\Delta_{-} \Delta_{+} f\left(\max \left(u_{j}, \bar{u}\right)\right)\right] \\
=\sum\left[\rho(j \Delta x) u_{j} \Delta_{+} f\left(u_{j}\right)+\left(\Delta_{+} \rho(j \Delta x) u_{j}\right) \Delta_{+} f\left(\max \left(u_{j}, \bar{u}\right)\right)\right] \\
=\sum \rho(j \Delta x)\left[u_{j} \Delta_{+} f\left(u_{j}\right)+\left(\Delta_{+} u_{j}\right) \Delta_{+} f\left(\max \left(u_{j}, \bar{u}\right)\right)\right] \\
\quad+\sum\left(\Delta_{+} \rho(j \Delta x)\right) u_{j+1} \Delta_{+} f\left(\max \left(u_{j}, \bar{u}\right)\right) \\
=\sum \rho(j \Delta x) \int_{u_{j}}^{u_{j+1}}\left(u_{j}+\left(\Delta_{+} u_{j}\right) \chi(s)\right) f^{\prime}(s) d s \\
\quad+\sum\left(\Delta_{+} \rho(j \Delta x)\right) u_{j+1} \int_{u_{j}}^{u_{j+1}} \chi(s) f^{\prime}(s) d s .
\end{aligned}
$$

Each step is justified as follows: We use the equality $f\left(u_{j}\right)=f\left(\min \left(u_{j}, \bar{u}\right)\right)+$ $f\left(\max \left(u_{j}, \bar{u}\right)\right)-f(\bar{u})$ in line 2 , summation by parts for line 3 , the equality $\Delta_{+}\left(u_{j} v_{j}\right)=$ $\left(\Delta_{+} u_{j}\right) v_{j}+\left(\Delta_{+} v_{j}\right) u_{j+1}$ for line 4 , and the fact that if $f^{\prime \prime}>0$ then

$$
f\left(\max \left(u_{j}, \bar{u}\right)\right)-f(\bar{u})=\int_{\bar{u}}^{u_{j}} f^{\prime}(s) \chi(s) d s
$$

to justify the last line.

Next we add to the above equality

$$
\begin{aligned}
0 & =-\sum_{j} \Delta_{+}\left(\rho(j \Delta x) \int_{0}^{u_{j}} s f^{\prime}(s) d s\right) \\
& =-\sum \rho(j \Delta x) \int_{u_{j}}^{u_{j+1}} s f^{\prime}(s) d s-\sum\left(\Delta_{+} \rho(j \Delta x)\right) \int_{0}^{u_{j+1}} s f^{\prime}(s) d s \\
& =-\sum \rho(j \Delta x) \int_{u_{j}}^{u_{j+1}} s f^{\prime}(s) d s-\sum\left(\Delta_{-} \rho(j \Delta x)\right) \int_{0}^{u_{j}} s f^{\prime}(s) d s .
\end{aligned}
$$


The result is now immediate.

Proof of Lemma 2.2. We have

$$
\begin{aligned}
& \sum \rho(j \Delta x, k \Delta y) u_{j k} \Delta_{+}^{y} v_{j k}=-\sum\left(\Delta_{-}^{y} \rho(j \Delta x, k \Delta y) u_{j k}\right) v_{j k} \\
& \quad=-\sum\left(\Delta_{-}^{y} \rho(j \Delta x, k \Delta y) u_{j k}\right) v_{j k}-\sum \rho(j \Delta x,(k-1) \Delta y)\left(\Delta_{-}^{y} u_{j k}\right) v_{j k} \\
& \quad=-\sum\left(\Delta_{-}^{y} \rho(j \Delta x, k \Delta y)\right) u_{j k} v_{j k}-\frac{\Delta y}{\Delta x} \sum \rho(j \Delta x,(k-1) \Delta y)\left(\Delta_{-}^{x} v_{j k}\right) v_{j k} .
\end{aligned}
$$

We add to this

$$
0=\frac{\Delta y}{2 \Delta x} \sum \Delta_{-}^{x} \rho(j \Delta x,(k-1) \Delta y) v_{j k}^{2}
$$

$$
\begin{aligned}
= & \frac{\Delta y}{2 \Delta x} \sum \rho(j \Delta x,(k-1) \Delta y) \Delta_{-}^{x} v_{j k}^{2} \\
& +\frac{\Delta y}{2 \Delta x} \sum\left(\Delta_{-}^{x} \rho(j \Delta x,(k-1) \Delta y)\right) v_{j-1, k}^{2} .
\end{aligned}
$$

Using the fact that

$$
-\left(\Delta_{-}^{x} v_{j k}\right) v_{j k}+1 / 2 \Delta_{-}^{x} v_{j k}^{2}=-1 / 2\left(\Delta_{-}^{x} v_{j k}\right)^{2},
$$

we arrive at

$$
\begin{aligned}
& \sum \rho(j \Delta x, k \Delta y) u_{j k} \Delta_{+}^{y} v_{j k} \\
& =-\sum\left(\Delta_{-}^{y} \rho(j \Delta x, k \Delta y)\right) u_{j k} v_{j k}-\frac{1}{2} \frac{\Delta y}{\Delta x} \sum \rho(j \Delta x,(k-1) \Delta y)\left(\Delta_{-}^{x} v_{j k}\right)^{2} \\
& \quad+\frac{\Delta y}{2 \Delta x} \sum\left(\Delta^{x} \rho(j \Delta x,(k-1) \Delta y)\right) v_{j-1, k}^{2} .
\end{aligned}
$$

3. Stability and the Entropy Inequality for the Time-Dependent Scheme. In this section we shall consider an initial-boundary value problem for the time-dependent partial differential equation (1.2)

$$
\Phi_{x t}=\left(K \Phi_{x}-1 / 2(\gamma+1) \Phi_{x}^{2}\right)_{x}+\Phi_{y y} .
$$

(For simplicity we normalize and remove the factor of 2 appearing in (1.2).)

We shall solve this for $t>0$, and for convenience only, in the square in $R^{2}$

$$
\Omega=\{x, y \mid 0<x<1,0<y<1\}
$$

we prescribe the following initial and boundary conditions

(a) $\Phi(x, y, 0)=\Phi_{0}(x, y)$,

(b) $\Phi(0, y, t)=g(y, t)$,

$$
\begin{aligned}
& \Phi_{x}(0, y, t)=p(y, t), \\
& \Phi_{x}(1, y, t)=h(y, t), \\
& \Phi_{y}(x, 0, t)=G(x, t), \\
& \Phi_{y}(x, 1, t)=H(x, t) .
\end{aligned}
$$


It is by no means certain that this problem is well posed. An analysis of the simplified $y$-independent problem follows.

In one space dimension we have

(a) $u_{t}=-(f(u))_{x}, \quad 0<x<1$,

(b) $u(x, 0)=u_{0}(x)$,

(c) $u(0, t)=p(t)$

$$
u(1, t)=h(t) \text {. }
$$

For smooth initial data the problem is well posed for small time if and only if $p(t)>$ $\bar{u}>h(t)$, i.e. supersonic in inflow, subsonic outflow.

Moreover, if the solution to this equation converges as $t \rightarrow \infty$ to a time-independent solution of (3.3), (assuming $p$ and $h$ are time-independent), we have

$$
\begin{aligned}
0 & =\frac{\partial}{\partial t} \int_{0}^{1} u d x=-\int_{0}^{1}(f(u))_{x} d x=-f(u(1))+f(u(0)) \\
& =-f(h)+f(p) .
\end{aligned}
$$

Thus, we must choose $h$ and $p$ so that

$$
p>\bar{u}>h, \quad f(p)=f(h) .
$$

The situation is much more complicated in two space dimensions. Nevertheless, we shall establish rigorous a priori estimates to a difference approximation to (3.1), (3.2) based on the method of fractional steps and our version of the Cole-Murman differencing in the space variables.

We set up a lattice

$$
\begin{aligned}
x_{j}=j \Delta x, \quad y_{k}=k \Delta y, \quad t_{n}=\sum_{0}^{n-1} \Delta t_{j}, \\
j=-1,0, \ldots, N ; k=-1,0, \ldots, M+1,
\end{aligned}
$$

with $N \Delta x=1=M \Delta y$.

We are seeking a discrete potential function $\varphi_{j k}^{n}$ which is supposed to approximate $\Phi\left(j \Delta x, k \Delta y, t_{n}\right)$.

Using backwards differencing, we again define

$$
u_{j k}^{n}=D_{-}^{x} \varphi_{j k}^{n}, \quad v_{j k}^{n}=D_{-}^{y} \varphi_{j k}^{(n)}, \quad j=0,1, \ldots, N, k=0,1, \ldots, M+1 .
$$

We prescribe discrete initial data $\varphi_{j k}^{0}=\Phi_{0}(j \Delta x, k \Delta y)$ and the difference scheme which we set up is the following:

We first advance one half step via the difference scheme

$$
u_{j k}^{n+(1 / 2)}=u_{j k}^{n}-\lambda_{n}^{(x)} \Delta_{+}^{x} f\left(\min \left(u_{j k}^{n}, \bar{u}\right)\right)-\lambda_{n}^{(x)} \Delta_{-}^{x} f\left(\max \left(u_{j k}^{n}, \bar{u}\right)\right)
$$

where $\lambda_{n}^{(x)}=\Delta t_{n} / \Delta x, j=1,2, \ldots, N-1 ; k=0,1, \ldots, M$.

The boundary conditions are

$$
u_{0 k}^{n+(1 / 2)}=p\left(k \Delta y, t_{n+1}\right), \quad u_{N k}^{n+(1 / 2)}=h\left(k \Delta y, t_{n+1}\right) .
$$


Next, we recover

$$
\varphi_{j k}^{n+(1 / 2)}=g\left(k \Delta y, t_{n+1}\right)-\Delta x p\left(k \Delta y, t_{n+1}\right)+\sum_{r=0}^{j} u_{r k}^{n+(1 / 2)} \Delta x
$$

(so $\left.D_{-}^{x} \varphi_{j k}^{n+(1 / 2)}=u_{j k}^{n+(1 / 2)}\right)$.

Then we advance to time $t_{n}$ via the linear Crank-Nicolson type of $y$ differencing

$$
u_{j k}^{n+1}=u_{j k}^{n+(1 / 2)}+1 / 2 \lambda_{n}^{(y)} \Delta_{+}^{y}\left(v_{j k}^{n+1}+v_{j k}^{n+(1 / 2)}\right) \text { where } \lambda_{n}^{(y)}=\Delta t_{n} / \Delta y,
$$

$$
j=1,2, \ldots, N-1 ; k=0,1, \ldots, M \text {. }
$$

The boundary conditions are

$$
\begin{aligned}
& u_{0 k}^{n+1}=p\left(k \Delta y, t_{n+1}\right), \\
& \varphi_{0 k}^{n+1}=g\left(k \Delta y, t_{n+1}\right), \quad k=0,1, \ldots, M, \\
& u_{N k}^{n+1}=h\left(k \Delta y, t_{n+1}\right), \\
& v_{j 1}^{n+1}=G\left(j \Delta x, t_{n+1}\right), \\
& v_{j, M+1}^{n+1}=H\left(j \Delta x, t_{n+1}\right), \quad j=1, \ldots, N-1 .
\end{aligned}
$$

The iteration is then repeated.

We shall prove that this procedure generates an $L^{2}$ stable scheme under the Courant condition

$$
\max _{u_{j k}^{n}} \lambda_{n}^{(x)}\left|f^{\prime}\left(u_{j k}^{n}\right)\right|=\epsilon_{0}<1 / 3 .
$$

We shall show that this implicit linear system is uniquely invertible for $\varphi_{j k}^{n+1}$. Moreover, the inversion of the implicit linear system (3.10) for $\varphi_{j k}^{n+1}$ is particularly simple and is described midway through the proof of Lemma 3.2 below.

In order to invert the time-independent discrete system of equations (2.8) in the square, we can iterate (3.7), (3.10) and let $n \rightarrow \infty$. Presumably this procedure converges to a steady solution. The numerical evidence of Section 5 verifies this conjecture.

It is easy to see that (3.7) and (3.10) are first order accurate as operators on $\varphi_{j k}^{n}$. However, if there is convergence to a time-independent solution, then (3.7), (3.10) has the same spatial accuracy as (2.8), i.e. second order in subsonic and first order in supersonic regions.

We define the following discrete $L^{2}$ norms

$$
\begin{gathered}
\|u\|^{2}=\sum_{j=0}^{N} \sum_{k=0}^{M} u_{j k}^{2} \Delta x \Delta y, \quad\|u\|_{\Delta x}^{2}=\sum_{j=0}^{N} u_{j}^{2} \Delta x, \\
\|u\|_{\Delta y}^{2}=\sum_{k=0}^{M} u_{k}^{2} \Delta y .
\end{gathered}
$$

We may now state the main theorems of this section.

THEOREM 3.1. Let $\varphi_{j k}^{n}, u_{j k}^{n}, v_{j k}^{n}$ be defined as in (3.6)-(3.11) with the Courant condition (3.11). Then we have the following $L^{2}$ stability result 


$$
\begin{aligned}
\left\|u^{n}\right\|^{2}-\left\|u^{0}\right\|^{2} \leqslant & 2 \sum_{\mu=1}^{n} \Delta x\left(\left\|p\left(\cdot, t_{\mu}\right)\right\|_{\Delta y}^{2}+\left\|h\left(\cdot, t_{\mu}\right)\right\|_{\Delta y}^{2}\right) \\
& +\sum_{\mu=1}^{n} \Delta t_{\mu-1}\left\|D_{-1}^{y} y\left(\cdot, t_{\mu}\right)\right\|_{\Delta y}^{2} \\
& +4 \sum_{\mu=1}^{n} \frac{\left(\Delta t_{\mu-1}\right)^{2}}{\Delta y}\left(\left\|G\left(\cdot, t_{\mu}\right)\right\|_{\Delta x}^{2}+\left\|H\left(\cdot, t_{\mu}\right)\right\|_{\Delta x}^{2}\right) \\
\text { (3.14) } & +2 \sum_{\mu=0}^{n-1} \Delta t_{\mu} \sum_{j=0}^{m}\left(\frac{K}{2} h^{2}\left(j \Delta y, t_{\mu}\right)-\frac{(\gamma+1)}{3} h^{3}\left(j \Delta y, t_{\mu}\right)\right) \Delta y \\
& -2 \sum_{\mu=0}^{n-1} \Delta t_{\mu} \sum_{j=0}^{m}\left(\frac{K}{2} p^{2}\left(j \Delta y, t_{\mu}\right)-\frac{(\gamma+1)}{3} p^{3}\left(j \Delta y, t_{\mu}\right)\right) \Delta y .
\end{aligned}
$$

Moreover, if we know that for each $n$

$$
u_{N k}^{n}, u_{N-1, k}^{n} \leqslant \bar{u} \leqslant u_{0 k}^{n}, u_{1 k}^{n},
$$

ie. supersonic inflow, subsonic outflow, the first two terms on the right in (3.14) may be replaced by a telescoping series in $\mu$, summing to

$$
2 \Delta x\left(\left\|p\left(\cdot, t_{n}\right)\right\|^{2}-\|p(\cdot, 0)\|^{2}+\left\|h\left(\cdot, t_{n}\right)\right\|^{2}-\|h(\cdot, 0)\|^{2}\right) .
$$

We also prove that the time-dependent entropy condition is satisfied by limit solutions (3.6)-(3.11) under the Courant condition (3.12).

THEOREM 3.2. Let $\varphi_{j k}^{n}$ be defined as above and suppose $\varphi_{j k}^{n}, u_{j k}^{n}, v_{j k}^{n}$ converge boundedly a.e. as $\Delta x, \Delta y, \Delta t_{n} \rightarrow 0$ to $\Phi, u$, and $v$. Then $\Phi$ is a weak solution of (3.1) which satisfies the entropy inequality: For any $\rho \in C_{0}^{1}, \rho \geqslant 0 . \rho \equiv 0$ near the space boundary and near $t=0$

$$
\iiint\left[\rho_{t} \frac{\Phi_{x}^{2}}{2}+\rho_{x} F\left(\Phi_{x}, \Phi_{y}\right)+\rho_{y} G\left(\Phi_{x}, \Phi_{y}\right)\right] d x d y d t \geqslant 0,
$$

where $F$ and $G$ are defined in (2.12).

We shall prove these theorems with the help of two technical lemmas.

LEMMA 3.1. For $f(u)=-K u+(\gamma+1) u^{2} / 2, \bar{u}=K /(\gamma+1)$, solutions of the difference scheme

$$
\begin{aligned}
& u_{j}^{n+(1 / 2)}=u_{j}^{n}-\lambda_{n} \Delta_{+} f\left(\min \left(u_{j}^{n}, \bar{u}\right)\right)-\lambda_{n} \Delta_{-} f\left(\max \left(u_{j}^{n}, \bar{u}\right)\right), \\
& j=1, \ldots, N-1,
\end{aligned}
$$

under the Courant condition (3.13) with boundary conditions

$$
\begin{array}{ll}
u_{0}^{n+(1 / 2)}=p\left(t_{n+1}\right), & u_{0}^{n}=p\left(t_{n}\right), \\
u_{N}^{n+(1 / 2)}=h\left(t_{n+1}\right), & u_{N}^{n}=h\left(t_{n}\right),
\end{array}
$$


satisfy the $L^{2}$ estimate

$$
\begin{aligned}
\frac{\left\|u^{n+(1 / 2)}\right\|_{\Delta x}^{2}-\left\|u^{n}\right\|_{\Delta x}^{2}}{2 \Delta t_{n}} \leqslant & \frac{1}{\lambda_{n}} h^{2}\left(t_{n+1}\right)+K \frac{h^{2}\left(t_{n+1}\right)}{2}-(\gamma+1) \frac{h^{3}\left(t_{n}\right)}{3} \\
& +\frac{1}{\lambda_{n}} p^{2}\left(t_{n+1}\right)-\frac{K}{2} p^{2}\left(t_{n}\right)+(\gamma+1) \frac{p^{3}\left(t_{n}\right)}{3}
\end{aligned}
$$

and if $u_{N}^{n}, u_{N-1}^{n} \leqslant \bar{u} \leqslant u_{0}^{n}, u_{1}^{n}$, we may subtract $\left(h^{2}\left(t_{n}\right)+p^{2}\left(t_{n}\right)\right) / \lambda_{n}$ from the right side above. Moreover, if $\rho(x, t) \geqslant 0, \rho \equiv 0$ near $x=0$ and $x=1$, we have the inequality

$$
\begin{aligned}
& \sum_{j} \Delta x {\left[\rho\left(j \Delta x, t_{n}\right) \frac{\left(u_{j}^{n+(1 / 2)}\right)^{2}-\left(u_{j}^{n}\right)^{2}}{2 \Delta t_{n}}\right.} \\
&\left.-\left(\frac{\Delta_{-}^{x} \rho\left(j \Delta x, t_{n}\right)}{\Delta x}\right)\left(-K \frac{\left(u_{j}^{n}\right)^{2}}{2}+(\gamma+1) \frac{\left(u_{j}^{n}\right)^{3}}{3}\right)\right] \\
& \leqslant-\sum_{j} \Delta x {\left[\frac { ( \Delta _ { + } ^ { x } \rho ( j \Delta x , t _ { n } ) ) } { \Delta x } \left(u_{j+1}^{n} \int_{u_{j}^{n}}^{u_{j+1}^{n}} \chi(s) f^{\prime}(s) d s\right.\right.} \\
&\left.\left.-\lambda_{n}\left(\Delta_{+}^{x} f\left(\max \left(u_{j}^{n}, \bar{u}\right)\right)\right)^{2}\right)\right] .
\end{aligned}
$$

LEMMA 3.2. The difference scheme

$$
u_{j k}^{n+1}=u_{j k}^{n+(1 / 2)}+\lambda_{n}^{(y)} \Delta_{+}^{y} v_{j k}^{\tilde{n}}
$$

to be solved on the lattice $j=1, \ldots, N-1, k=0,1, \ldots, M$, with boundary conditions (3.11) at both time levels $n+1$ and $n+(1 / 2)$ satisfies the $L^{2}$ inequality

$$
\begin{aligned}
& \frac{\left\|u^{n+1}\right\|^{2}-\left\|u^{n+(1 / 2)}\right\|^{2}}{2 \Delta t_{n}} \\
& \quad \leqslant \frac{1}{2}\left\|D_{-}^{y} g\left(\cdot, t_{n+1}\right)\right\|_{\Delta x}^{2}+2 \frac{\Delta t_{n}}{\Delta y}\left(\left\|G\left(\cdot, t_{n+1}\right)\right\|_{\Delta x}^{2}+\left\|H\left(\cdot, t_{n+1}\right)\right\|_{\Delta x}^{2}\right) .
\end{aligned}
$$

Here and in what follows $v^{\tilde{n}}=1 / 2\left(v^{n+1}+v^{n+(1 / 2)}\right)$ etc.

Moreover, if $\rho(x, y, t) \geqslant 0, \rho \equiv 0$ near the boundaries $x=0, x=1, y=0$ and $y=1$, we have the equality

$$
\begin{aligned}
\sum_{j, k} \rho\left(j \Delta x, k \Delta y, t_{n}\right)\left[\frac{\left(u_{j k}^{n+1}\right)^{2}-\left(u_{j k}^{n+(1 / 2)}\right)^{2}}{2 \Delta t_{n}}\right] \Delta x \Delta y \\
+\sum_{j, k}\left(\Delta_{-}^{y} \rho\left(j \Delta x, k \Delta y, t_{n}\right)\right) u_{j k}^{n} v_{j k}^{n} \Delta x \\
\quad-\frac{1}{2} \sum_{j, k}\left(\Delta_{-}^{x} \rho\left(j \Delta x,(k-1) \Delta y, t_{n}\right)\right)\left(v_{j-1, k}^{\tilde{n}}\right)^{2} \Delta y \\
=-\frac{1}{2} \sum_{j, k} \rho\left(j \Delta x,(k-1) \Delta y, t_{n}\right)\left(\Delta_{-}^{x} v_{j k}^{\tilde{n}}\right)^{2} \Delta y .
\end{aligned}
$$


Remark 1. The difference scheme (3.21) is unconditionally stable, i.e. $\lambda_{n}^{(x)}$, $\lambda_{n}^{(y)}$ can be chosen arbitrarily. For convenience we take $\Delta t_{n}$ at this half step to be the same as for the first half step.

Remark 2. Some of the boundary conditions of (3.11) are not needed for the second half step. In particular, the values of $\varphi_{j k}$ at $j=-1$ are never used. However, we need them for the first half step if the inflow is supersonic.

We shall prove these technical lemmas below. We may now use them for the following.

Proof of Theorem 3.1. For $u_{j k}^{n}$ solving (3.17), we multiply (3.19) by $2 \Delta y \Delta t_{n}$ and $\operatorname{sum} k=0, \ldots, M$. Next multiply (3.22) by $2 \Delta t_{n}$ and add to (3.22). We then sum from $\mu=0$ to $\mu=n$, the result is immediate.

Proof of Theorem 3.2. We add (3.20) for the $y$-dependent case to (3.23), then sum over $n$. The result is immediate from the Lebesgue dominated convergence theorem.

We must now merely prove Lemmas 3.1 and 3.2.

Proof of Lemma 3.1. Let $u_{j}^{n+(1 / 2)}=w_{j}, u_{j}^{n}=u_{j}, \lambda_{n}=\lambda$. We have, after multiplying by $\left(w_{j}+u_{j}\right) / 2 \lambda$ and adding

$$
\begin{aligned}
\|w\|_{\Delta x}^{2}-\|u\|_{\Delta x}^{2}= & \left(u_{j}-\frac{\lambda \Delta_{+} f\left(\min \left(u_{j}, \bar{u}\right)\right)}{2}-\frac{\lambda \Delta_{-} f\left(\max \left(u_{j}, \bar{u}\right)\right)}{2},\right. \\
& \left.-\lambda \Delta_{+} f\left(\min \left(u_{j}, \bar{u}\right)\right)-\lambda_{-} f\left(\max \left(u_{j}, \bar{u}\right)\right)\right)_{\Delta x},
\end{aligned}
$$

or

$$
\begin{aligned}
\frac{\|w\|_{\Delta x}^{2}-\|u\|_{\Delta x}^{2}}{2 \Delta t}=- & \sum_{j=1}^{N-1} u_{j}\left(\Delta_{+} f\left(\min \left(u_{j}, \bar{u}\right)\right)+\Delta_{-} f\left(\max \left(u_{j}, \bar{u}\right)\right)\right) \\
& +\frac{\lambda}{2} \sum_{j=1}^{N-1}\left(\Delta_{+} f\left(\min \left(u_{j}, \bar{u}\right)\right)+\Delta_{-}\left(\max \left(u_{j}, \bar{u}\right)\right)\right)^{2} \\
& +\frac{1}{2 \lambda}\left(\left|w_{N}\right|^{2}-\left|u_{N}\right|^{2}\right)+\frac{1}{2 \lambda}\left(\left|w_{0}\right|^{2}-\left|u_{0}\right|^{2}\right) .
\end{aligned}
$$

It is a simple matter to go through the proof of Lemma 2.1 for $\rho \equiv 1$ and take boundary terms into account to arrive at

$$
\begin{aligned}
-\sum_{j=1}^{N-1} u_{j}\left(\Delta_{+} f\left(\min \left(u_{j}, \bar{u}\right)\right)+\Delta_{-} f\left(\max \left(u_{j}, \bar{u}\right)\right)\right) \\
=\sum_{j=0}^{N-1} \int_{u_{j}}^{u_{j+1}}\left(s-u_{j}-\left(\Delta_{+} u_{j}\right) \chi(s)\right) f^{\prime}(s) d s+u_{N} \Delta_{+} f\left(\max \left(u_{n-1}, \bar{u}\right)\right) \\
\quad-\int_{u_{0}}^{u_{N}} s f^{\prime}(s) d s+u_{0} \Delta_{+} f\left(\min \left(u_{0}, \bar{u}\right)\right) .
\end{aligned}
$$

Recall $\chi(s)$ was defined in the statement of Lemma 2.1 . 
It follows from Schwarz' inequality that

$$
\begin{aligned}
& \frac{\lambda}{2} \sum_{j=1}^{N-1}\left(\Delta_{+} f\left(\min \left(u_{j}, \bar{u}\right)\right)+\Delta_{-} f\left(\max \left(u_{j}, \bar{u}\right)\right)\right)^{2} \\
& \leqslant \lambda \sum_{j=0}^{N-1}\left(\Delta_{+} f\left(\min \left(u_{j}, \bar{u}\right)\right)\right)^{2}+\lambda \sum_{j=0}^{N-1}\left(\Delta_{+} f\left(\max \left(u_{j}, \bar{u}\right)\right)\right)^{2} \\
&-\lambda\left(\Delta_{+} f\left(\min \left(u_{0}, \bar{u}\right)\right)\right)^{2}-\lambda\left(\Delta_{+} f\left(\max \left(u_{N-1}, \bar{u}\right)\right)\right)^{2}
\end{aligned}
$$

Adding (3.26) to (3.27) gives us

$$
\begin{aligned}
\frac{\|w\|_{\Delta x}^{2}-\|u\|_{\Delta x}^{2}}{2 \Delta t} \leqslant & \sum_{j=0}^{N-1}\left[\int_{u_{j}}^{u_{j+1}}\left(s-u_{j}-\Delta_{+} u_{j} \chi(s)\right) f^{\prime}(s) d s\right. \\
& \left.\quad+\lambda\left(\Delta_{+} f\left(\min \left(u_{j}, \bar{u}\right)\right)\right)^{2}+\lambda\left(\Delta_{+} f\left(\max \left(u_{j}, \bar{u}\right)\right)\right)^{2}\right] \\
& +u_{N} \Delta_{+} f\left(\max \left(u_{N-1}, \bar{u}\right)\right)-\lambda\left(\Delta_{+} f\left(\max \left(u_{N-1}, \bar{u}\right)\right)\right)^{2} \\
& -\int_{0}^{u_{N}} s f^{\prime}(s) d s+\frac{1}{2 \lambda}\left(p^{2}\left(t_{n+1}\right)-p^{2}\left(t_{n}\right)\right) \\
& +u_{0} \Delta_{+} f\left(\min \left(u_{0}, \bar{u}\right)\right)-\lambda\left(\Delta_{+} f\left(\min \left(u_{0}, \bar{u}\right)\right)\right)^{2} \\
& +\int_{0}^{u_{0}} s f^{\prime}(s) d s+\frac{1}{2 \lambda}\left(h^{2}\left(t_{n+1}\right)-h^{2}\left(t_{n}\right)\right) .
\end{aligned}
$$

We wish to estimate the term under the $\Sigma$ sign

$$
\begin{aligned}
A_{j, j+1}= & \int_{u_{j}}^{u_{j+1}}\left(s-u_{j}-\left(\Delta_{+} u_{j}\right) \chi(s)\right) f^{\prime}(s) d s+\lambda\left(\Delta_{+} f\left(\min \left(u_{j}, \bar{u}\right)\right)\right)^{2} \\
& +\lambda\left(\Delta_{+} f\left(\max \left(u_{j}, \bar{u}\right)\right)\right)^{2}
\end{aligned}
$$

If $u_{j+1}, u_{j} \geqslant \bar{u}$, a straightforward calculation gives us

$$
\begin{aligned}
A_{j, j+1}=\frac{\left(u_{j+1}-u_{j}\right)^{2}}{2}([K- & \left.(\gamma+1)\left(\frac{2}{3} u_{j}+\frac{1}{3} u_{j+1}\right)\right] \\
& \left.+2 \lambda\left[-K+(\gamma+1) \frac{\left(u_{j+1}+u_{j}\right)}{2}\right]^{2}\right) \leqslant 0,
\end{aligned}
$$

if $\epsilon_{0} \leqslant 1 / 3$.

Similarly, if $u_{j+1}, u_{j} \leqslant \bar{u}$,

$$
\begin{aligned}
A_{j, j+1}=\frac{\left(u_{j+1}-u_{j}\right)^{2}}{2}([- & \left.+(\gamma+1)\left(\frac{1}{3} u_{j}+\frac{2}{3} u_{j+1}\right)\right] \\
& \left.+2 \lambda\left[-K+(\gamma+1) \frac{\left(u_{j+1}+u_{j}\right)}{2}\right]^{2}\right) \leqslant 0,
\end{aligned}
$$

if $\epsilon_{0} \leqslant 1 / 3$. 
Next, suppose $u_{j+1} \geqslant \bar{u} \geqslant u_{j}$,

$$
\begin{aligned}
A_{j, j+1}= & \left(u_{j}-\bar{u}\right)^{2}\left[\left(\frac{\gamma+1}{6}\right)\left(u_{j}-\bar{u}\right)+\lambda\left(K-(\gamma+1)\left(\frac{u_{j}+\bar{u}}{2}\right)\right)^{2}\right] \\
& +\left(u_{j+1}-\bar{u}\right)^{2}\left[\left(\frac{\gamma+1}{6}\right)\left(\bar{u}-u_{j+1}\right)+\lambda\left(K-(\gamma+1)\left(\frac{u_{j}+\bar{u}}{2}\right)\right)^{2}\right] \\
= & \left(u_{j}-\bar{u}\right)^{2}\left[\left(\frac{-K+(\gamma+1) u_{j}}{6}\right)+\lambda\left(\frac{K-(\gamma+1) u_{j}}{2}\right)^{2}\right] \\
& +\left(u_{j+1}-\bar{u}\right)^{2}\left[\left(\frac{K-(\gamma+1) u_{j+1}}{6}\right)+\lambda\left(\frac{K-(\gamma+1) u_{j+1}}{2}\right)^{2}\right] \leqslant 0,
\end{aligned}
$$

if $\epsilon_{0}<1 / 3$; and finally, if $u_{j+1} \leqslant \bar{u} \leqslant u_{j}$,

$$
\begin{aligned}
A_{j, j+1}= & \frac{1}{2}\left(u_{j+1}-u_{j}\right)^{2}\left(-\frac{2}{3} K+\frac{2}{3}(\gamma+1) u_{j+1}+\frac{2}{3} K-\frac{2}{3}(\gamma+1) u_{j}\right) \\
& -\frac{(\gamma+1)}{6}\left(\bar{u}-u_{j+1}\right)^{3}+\frac{(\gamma+1)}{6}\left(\bar{u}-u_{j}\right)^{3} \\
& +\lambda\left(u_{j}-\bar{u}\right)^{2}\left[K-(\gamma+1)\left(\frac{u_{j}+\bar{u}}{2}\right)\right]^{2} \\
& +\lambda\left(u_{j+1}-\bar{u}\right)^{2}\left[K-(\gamma+1)\left(\frac{u_{j+1}+\bar{u}}{2}\right)\right]^{2} \\
\leqslant & \frac{1}{2}\left(u_{j+1}-\bar{u}\right)^{2}\left[-\frac{2}{3} K+(\gamma+1)\left(\frac{2}{3} u_{j+1}+\frac{1}{3} u_{j+1}-\frac{1}{3} \bar{u}\right)\right] \\
& +\frac{1}{2}\left(\bar{u}-u_{j}\right)^{2}\left[\frac{2}{3} K-(\gamma+1)\left(\frac{2}{3} u_{j+1}+\frac{1}{3} u_{j}-\frac{1}{3} \bar{u}\right)\right] \\
& +\lambda\left(u_{j}-\bar{u}\right)^{2}\left[K-(\gamma+1)\left(\frac{u_{j}+\bar{u}}{2}\right)\right]^{2} \\
& +\lambda\left(u_{j+1}-\bar{u}\right)^{2}\left[K-(\gamma+1)\left(\frac{u_{j+1}+\bar{u}}{2}\right)\right]^{2} \\
\leqslant & \frac{1}{2}\left(u_{j+1}-\bar{u}\right)^{2}\left[-K+(\gamma+1) u_{j+1}\right]+\frac{1}{2}\left(\bar{u}-u_{j}\right)^{2}\left[K-(\gamma+1) u_{j}\right] \\
& +\lambda\left(u_{j}-\bar{u}\right)^{2}\left[K-(\gamma+1)\left(\frac{u_{j}+\bar{u}}{2}\right)\right]^{2} \\
& +\lambda\left(u_{j+1}-\bar{u}\right)^{2}\left[K-(\gamma+1)\left(\frac{u_{j+1}+\bar{u}}{2}\right)\right]^{2} \leqslant 0, \\
& \\
&
\end{aligned}
$$


if $\epsilon_{0} \leqslant 1 / 2$. Thus, we have the "interior" dissipative estimate

$$
\sum_{j=0}^{N-1} A_{j, j+1} \leqslant 0
$$

with equality holding if and only if $u_{j}$ is constant. Applying Schwarz' inequality gives us

$$
\begin{gathered}
u_{N} \Delta_{+} f\left(\max \left(u_{N-1}, \bar{u}\right)\right)-\lambda\left(\Delta_{+} f\left(\max \left(u_{N-1}, \bar{u}\right)\right)\right)^{2} \leqslant \frac{1}{2 \lambda} u_{N}^{2}, \\
u_{0} \Delta_{+} f\left(\min \left(u_{0}, \bar{u}\right)\right)-\lambda\left(\Delta_{+} f\left(\min \left(u_{0}, \bar{u}\right)\right)\right)^{2} \leqslant \frac{1}{2 \lambda} u_{0}^{2}
\end{gathered}
$$

We may now add up all the boundary contributions, arriving at

$$
\begin{gathered}
\frac{\|w\|^{2}-\|u\|^{2}}{2 \Delta t} \leqslant \sum_{j=0}^{N-1} A_{j, j+1}+\frac{1}{\lambda} p^{2}\left(t_{n+1}\right)-\frac{K}{2} p^{2}\left(t_{n}\right)+(\gamma+1) \frac{p^{3}\left(t_{n}\right)}{3} \\
+\frac{1}{\lambda} h^{2}\left(t_{n+1}\right)+K \frac{h^{2}\left(t_{n}\right)}{2}-(\gamma+1) \frac{h^{3}\left(t_{n}\right)}{3}
\end{gathered}
$$

thus proving estimate (3.19).

In order to prove the discrete entropy inequality, we again follow the proof of Lemma 2.1 , after multiplying by $\rho\left(j \Delta x, t_{n}\right)\left(u_{j}^{n+1}-u_{j}^{n}\right) / 2 \Delta t_{n}$. It is a straightforward calculation to show

$$
\begin{aligned}
\sum_{j}\left[\frac{\rho\left(j \Delta x, t_{n}\right)\left[\left(u_{j}^{n+1}\right)^{2}-\left(u_{j}^{n}\right)^{2}\right]}{2 \Delta t}\right] & \\
& -\frac{\left(\Delta_{-}^{x} \rho\left(j \Delta x, \Delta t_{n}\right)\right)}{\Delta t}\left(\frac{-K\left(u_{j}^{n}\right)^{2}}{2}+(\gamma+1) \frac{\left(u_{j}^{n}\right)^{3}}{2}\right)_{\Delta x}
\end{aligned}
$$

$$
\begin{aligned}
= & \sum_{j} \frac{1}{\Delta x} \rho\left(j \Delta x, t_{n}\right) A_{j, j+1} \Delta x \sum_{j} \frac{\left(\Delta_{+}^{x} \rho\left(j \Delta x, t_{n}\right)\right)}{\Delta x} u_{j+1} \int_{u_{j}}^{u_{j+1}} \chi(s) f^{\prime}(s) d s \Delta x \\
& +\lambda \sum_{j} \frac{\Delta_{+}^{x} \rho\left(j \Delta x, t_{n}\right)}{\Delta x}\left(\Delta_{+} f\left(\max \left(u_{j}, \bar{u}\right)\right)\right)^{2} \Delta x .
\end{aligned}
$$

The inequality follows from the fact that $A_{j, j+1} \leqslant 0$ and $\rho(x, t) \geqslant 0$.

Proof of Lemma 3.2. We multiply (3.21) by $u_{j k}^{n} \Delta x \Delta y$ and sum over $j=1, \ldots$, $N-1, k=0,1, \ldots, M$. We arrive at

$$
\frac{\left\|u^{n+1}\right\|^{2}-\left\|u^{n+(1 / 2)}\right\|^{2}}{2 \Delta t_{n}}=\sum_{k=0}^{M} \sum_{j=1}^{N-1} u_{j k}^{\tilde{n}}\left[v_{j, k+1}^{\tilde{n}}-v_{j k}^{\tilde{n}}\right] \Delta x .
$$

Following the proof of Lemma 2.2 for $\rho \equiv 1$, and taking boundaries into account, gives us 


$$
\begin{aligned}
\sum_{k=0}^{M} & \sum_{j=1}^{N-1} u_{j k}^{\tilde{n}}\left[v_{j, k+1}^{\tilde{n}}-v_{j k}^{\tilde{n}}\right] \Delta x \\
= & -\frac{1}{2} \sum_{j=1}^{N} \sum_{k=1}^{M+1}\left[v_{j k}^{\tilde{n}}-v_{j-1, k}^{\tilde{n}}\right]^{2} \Delta y-\frac{1}{2} \sum_{k=1}^{M+1}\left(v_{N k}^{\tilde{n}}\right)^{2} \Delta y+\frac{1}{2} \sum_{k=1}^{M+1}\left(v_{0 k}^{\tilde{n}}\right)^{2} \Delta y \\
& +\sum_{j=1}^{N-1} v_{j, M+1}^{\tilde{n}} u_{j, M+1}^{\tilde{n}} \Delta x-\sum_{j=1}^{N-1} v_{j 0}^{\tilde{n}} u_{j 0}^{\tilde{n}} \Delta x \\
& +\sum_{i=1}^{M+1} u_{N k}^{\tilde{n}}\left(v_{N k}^{\tilde{n}}-v_{N-1, k}^{\tilde{n}}\right) \Delta y .
\end{aligned}
$$

Suppose first that $v_{j, M+1}^{\tilde{n}} \equiv v_{j, 0}^{\tilde{n}} \equiv 0 \equiv G_{j}^{\tilde{n}} \equiv H_{j}^{\tilde{n}}$. Applying Schwarz' inequality gives us

$$
\begin{aligned}
-\frac{1}{2} \sum_{j=1}^{N} \sum_{k=1}^{M+1}\left[v_{j k}^{\tilde{n}}-u_{j-1, k}^{\tilde{n}}\right]^{2} \Delta y-\frac{1}{2} \sum_{k=1}^{M+1}\left(v_{N k}^{\tilde{n}}\right)^{2} \Delta y \\
+\sum_{k=1}^{M+1} v_{N, k}^{\tilde{n}}\left(v_{N k}^{\tilde{n}}-v_{N-1, k}^{\tilde{n}}\right) \Delta y \\
\leqslant-\frac{1}{2} \sum_{j=1}^{N-1} \sum_{k=1}^{M+1}\left[v_{j k}^{\tilde{n}}-v_{j-1, k}^{\tilde{n}}\right]^{2} \Delta y
\end{aligned}
$$

The estimate (3.22) follows in this special case.

In general, we write

$$
\varphi_{j k}^{m}=\varphi_{j k}^{m}(1)+\varphi_{j k}^{m}(2), \quad m=n+(1 / 2), n+1 .
$$

Here $\varphi_{j k}^{m}(1)$ satisfies the boundary conditions (3.11) except that $G_{j}^{m}$ and $H_{j}^{m}$ are replaced by zero. Thus, $\varphi_{j k}^{m}(2)$ satisfies (3.21) with the first three inhomogeneous functions taken to be identically zero.

We take

$$
\varphi_{j k}^{n+(1 / 2)}(1) \equiv \varphi_{j k}^{n+(1 / 2)} \text { for } 0 \leqslant k \leqslant M .
$$

Thus, $\varphi_{j k}^{n+1}(1), u_{j k}^{n+1}(1), v_{j k}^{n+1}(1)$ satisfy estimate (3.22) with $G$ and $H$ taken to be identically zero.

It follows that $\varphi_{j k}^{n+1}(2)=\psi_{j k}^{n+1}$ satisfies the following system of linear equations

$$
\begin{aligned}
\psi_{j k}^{n+1}-\frac{\mu_{n}}{2}\left[\psi_{j, k+1}^{n+1}-2 \psi_{j k}^{n+1}+\psi_{j, k-1}^{n+1}\right] & =\psi_{j-1, k}^{n+1}+\Delta y P_{j k}^{n}, \\
j & =1, \ldots, N-1 ; K=0,1, \ldots, M,
\end{aligned}
$$

where

$$
\begin{gathered}
P_{j k}^{n} \equiv 0, \quad \text { if } 0<k<m-1, \\
P_{j 0}^{n}=-1 / 2 \mu_{n} G_{j}^{n}, \quad P_{j M}^{n}=1 / 2 \mu_{n} H_{j}^{n},
\end{gathered}
$$

with $\mu_{n}=\Delta x \Delta t_{n} /(\Delta y)^{2}$. 
Let

$$
\Psi_{j}^{n+1}=\left(\begin{array}{c}
\psi_{j M}^{n+1} \\
\psi_{j, M-1}^{n+1} \\
\vdots \\
\psi_{j 0}^{n+1}
\end{array}\right)
$$

Then $\Psi_{j}^{n+1}$ solves

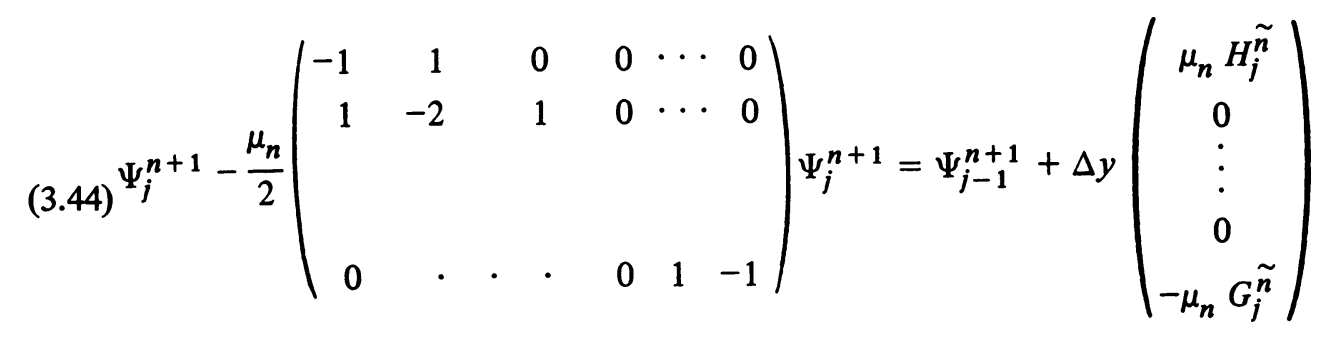

or (dropping the superscripts)

$$
T \Psi_{j}=\Psi_{j-1}+\Delta y F_{j}, \quad j=1, \ldots, N-1, \quad \Psi_{0}=0 .
$$

$T$ is a symmetric matrix which satisfies

$$
(\Psi, T \Psi)_{\Delta y}=\|\Psi\|_{\Delta y}^{2}+\frac{\mu_{n}}{2}\left\|\Delta_{-}^{y} \Psi\right\|_{\Delta y}^{2} .
$$

Thus, $T \geqslant I, T^{-1} \leqslant 1$. We have

$$
\Psi_{j}=\sum_{r=1}^{j} T^{-(j-r+1)} \Delta y F_{r}
$$

or

$$
\frac{\Psi_{j}-\Psi_{j-1}}{\Delta x}=\sum_{r=1}^{j-1} T^{-(j-r)}\left(T^{-1}-1\right) \frac{\Delta y}{\Delta x} F_{r}+T^{-1} \frac{\Delta y}{\Delta x} F_{j}
$$

Now, via a unitary change of variables, we have

$$
\begin{aligned}
& U^{*} T^{-(j-r)}\left(T^{-1}-I\right) U
\end{aligned}
$$

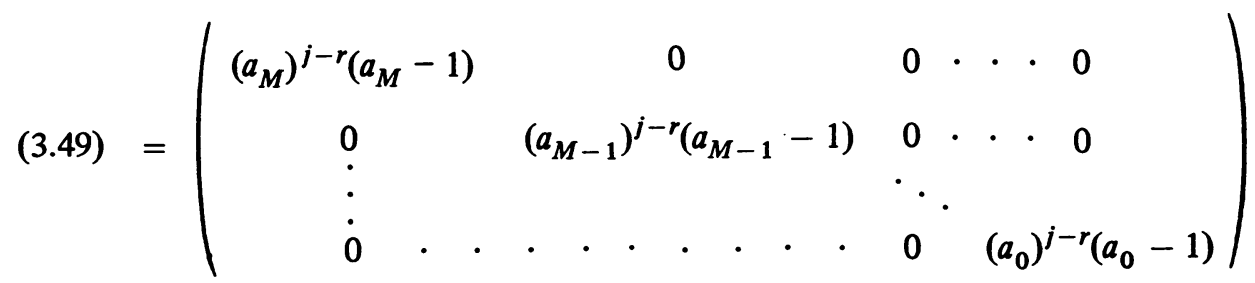

with $1 \geqslant a_{j} \geqslant 0$. It is thus easy to show that

(3.50) $\left\|\frac{\Psi_{j}-\Psi_{j-1}}{\Delta x}\right\|_{\Delta y}^{2} \leqslant 2\left(\frac{\Delta y}{\Delta x}\right)^{2}\left[\left\|F_{j}\right\|_{\Delta y}^{2}+\sup _{k} \sum_{r=1}^{j}\left\|F_{r}\right\|_{\Delta y}^{2} a_{k}^{j-r}\left(1-a_{k}\right)\right]$. 
Hence,

$$
\begin{gathered}
\left(\sum_{j=1}^{N-1}\left\|\frac{\Psi_{j}-\Psi_{j-1}}{\Delta x}\right\|_{\Delta y}^{2} \Delta x\right)^{1 / 2} \leqslant \sqrt{2} \frac{\Delta y}{\Delta x}\left(\sum_{r=1}^{N-1}\left\|F_{r}\right\|_{\Delta y}^{2} \Delta x\right)^{1 / 2} \\
\leqslant \sqrt{2} \frac{(\Delta y)^{3 / 2}}{\Delta x} \mu_{n}\left(\sum_{j=1}^{N-1}\left(H_{j}^{\tilde{n}}\right)^{2} \Delta x+\sum_{j=1}^{N-1}\left(G_{j}^{\tilde{n}}\right)^{2} \Delta x\right)^{1 / 2} .
\end{gathered}
$$

Thus,

$$
\frac{\left\|u_{(2)}^{n+1}\right\|^{2}}{2 \Delta t_{n}} \leqslant \frac{\Delta t_{n}}{\Delta y}\left(\sum_{j=1}^{N-1}\left(H_{j}^{\tilde{n}}\right)^{2} \Delta x+\sum_{r=1}^{N-1}\left(G_{j}^{\tilde{n}}\right)^{2} \Delta x\right) .
$$

Add (3.52) to (3.22) for $u^{n+1}(1)$ with $G \equiv H \equiv 0$. The result now follows from Schwarz' inequality.

The second part of this lemma follows easily from Lemma 2.2 after we multiply (3.21) by $\rho\left(j \Delta x, k \Delta y, t_{n}\right) u_{j k}^{\tilde{n}} \Delta x \Delta y$ and sum over $j, k$.

4. Stability and the Entropy Inequality for the Second Modification of the

Cole-Murman Scheme. In this section we analyze Cole-Murman type schemes (2.11) in some generality and point out how to modify the switch $\theta_{j k}$ such that limit solutions of the difference equation must satisfy the entropy inequality (2.12). We begin with the following lemma which is presented without proof. The proof is similar to that of Lemma 2.1 .

Lemma 4.1. Let $\rho(x) \in C_{0}^{1}$ with $\rho \geqslant 0$, and let $f^{\prime \prime}(u)>0$ with $f^{\prime}(\bar{u})>0$. Then

$$
\sum \rho(j \Delta x) u_{j}\left[\Delta_{+} f\left(u_{j}\right)-\Delta_{-} \theta_{j} \Delta_{+} f\left(u_{j}\right)\right]
$$

$$
\begin{aligned}
= & -\sum \rho(j \Delta x) \int_{u_{j}}^{u_{j+1}}\left[s-u_{j}-\left(\Delta_{+} u_{j}\right) \theta_{j}\right] f^{\prime}(s) d s \\
& -\sum\left(\Delta_{+} \rho(j \Delta x)\right) \int_{0}^{u_{j+1}} s f^{\prime}(s) d s+\sum\left(\Delta_{+} \rho(j \Delta x)\right) u_{j+1} \theta_{j} \Delta_{+} f\left(u_{j}\right) .
\end{aligned}
$$

Next we choose $\theta_{j}$ such that the inequality

$$
\int_{u_{j}}^{u_{j+1}}\left[\left(s-u_{j}\right)-\left(\Delta_{+} u_{j}\right) \theta_{j}\right] f^{\prime}(s) d s \leqslant 0
$$

is valid.

In the special case

$$
f(u)=-K u+\frac{(\gamma+1) u^{2}}{2}
$$

we have

$$
\begin{gathered}
\frac{\left(\Delta_{+} u_{j}\right)^{2}}{2}\left[\frac{2}{3} f^{\prime}\left(u_{j+1}\right)+\frac{1}{3} f^{\prime}\left(u_{j}\right)-\theta_{j}\left(\frac{1}{2} f^{\prime}\left(u_{j}\right)+\frac{1}{2} f^{\prime}\left(u_{j+1}\right)\right)\right] \leqslant 0, \text { or } \\
\frac{2}{3} f^{\prime}\left(u_{j+1}\right)+\frac{1}{3} f^{\prime}\left(u_{j}\right)-\theta_{j}\left(\frac{f^{\prime}\left(u_{j}\right)+f^{\prime}\left(u_{j+1}\right)}{2}\right) \leqslant 0 .
\end{gathered}
$$


Thus, if $u_{j}, u_{j+1} \leqslant \bar{u}$, we let $\theta_{j} \equiv 0$; and if $u_{j}, u_{j+1} \geqslant \bar{u}$ with at least one of $u_{j}$, $u_{j+1}$ strictly greater than $\bar{u}$, we let $\theta_{j} \equiv 1$.

So far this switch agrees with that of Cole-Murman. The difficulty occurs when $u_{j}, u_{j+1}$ are on opposite sides of $\bar{u}$. The C-M switch equals 1 (or 0 ) if $f^{\prime}\left(u_{j}\right)+$ $f^{\prime}\left(u_{j+1}\right)>0($ or $<0)$. As an example of the difficulties with this switch we might take

$$
\frac{f^{\prime}\left(u_{j}\right)+f^{\prime}\left(u_{j+1}\right)}{2}<0<\frac{2}{3} f^{\prime}\left(u_{j+1}\right)+\frac{1}{3} f^{\prime}\left(u_{j}\right)
$$

or

$$
f^{\prime}\left(u_{j+1}\right)>-\frac{1}{2} f^{\prime}\left(u_{j}\right)>+\frac{1}{2} f^{\prime}\left(u_{j+1}\right)>0
$$

so $u_{j+1}>\bar{u}>u_{j}$ in which case solutions with the C-M switch violate the inequality (4.3). This explains why C-M admits expansion shocks. In order to prevent these nonphysical solutions we let

$$
\theta_{j}\left(\frac{f^{\prime}\left(u_{j}\right)+f^{\prime}\left(u_{j+1}\right)}{2}\right)=\frac{2}{3} f^{\prime}\left(u_{j+1}\right)+\frac{1}{3} f^{\prime}\left(u_{j}\right)+a\left(\left|f^{\prime}\left(u_{j}\right)\right|+\left|f^{\prime}\left(u_{j+1}\right)\right|\right)
$$

for some $a>0$, when $\bar{u}$ lies in the open interval connecting $u_{j}$ and $u_{j+1}$. Since

$$
\theta_{j} \Delta_{+} f\left(u_{j}\right)=\left(\Delta_{+} u_{j}\right) \theta_{j}\left(\frac{f^{\prime}\left(u_{j}\right)+f^{\prime}\left(u_{j+1}\right)}{2}\right),
$$

-the resulting scheme (2.11) is well defined and first order accurate, although the coefficients are not continuous.

We can now easily prove the direct analog of Theorems $2.1,3.1$ and 3.2 for the C-M scheme with a switch of this sort.

THEOREM 4.1. Suppose $\varphi_{j k}$ is determined by (2.11), (4.4), and suppose $\varphi_{j k}$, $D_{-}^{x} \varphi_{j k}, D_{-}^{y} \varphi_{j k}$ converge boundedly a.e. as $\Delta x, \Delta y \rightarrow 0$ to $\Phi, \Phi_{x}$, and $\Phi_{y}$, respectively. Then, $\Phi$ is a weak solution of (2.1) which satisfies the entropy inequality (2.12).

The proof of the following lemma is now analogous to that of Lemma 3.1 using the previous analysis.

Lемма 4.1. For $f(u)=-K u+(\gamma+1) u^{2} / 2, \bar{u}=K /(\gamma+1)$, solutions of the difference scheme

$$
u_{j}^{n+1}=u_{j}^{n}-\lambda_{n} \Delta_{+} f\left(u_{j}^{n}\right)+\lambda_{n} \Delta_{-}\left(\theta_{j}^{n}\right) \Delta_{+} f\left(u_{j}^{n}\right),
$$

$$
j=1, \ldots, N-1, \text { with boundary conditions (3.8) }
$$

for $\theta_{j}^{n}$ defined in (4.4), and

$$
a=\frac{1}{4 \epsilon_{0}}-\frac{1}{6}
$$

satisfying the Courant condition 


$$
\max _{j, n}\left|\lambda_{n} f^{\prime}\left(u_{j}^{n}\right)\right|=\epsilon_{0} \leqslant 1 / 3,
$$

satisfy the $L^{2}$ estimates (3.19).

Moreover, if $\rho(x, t) \geqslant 0, \rho \equiv 0$ near $x=0$ and $x=1$, we have the inequality

$$
\begin{aligned}
& \sum_{j} \Delta x {\left[\rho\left(j \Delta x, t_{n}\right)\left(\frac{\left(u_{j}^{n+1}\right)^{2}-\left(u_{j}^{n}\right)^{2}}{2 \Delta t_{n}}\right)\right.} \\
&\left.\quad-\left(\frac{\Delta_{-}^{x} \rho\left(j \Delta x, t_{n}\right)}{\Delta x}\right)\left(\frac{-K\left(u_{j}^{n}\right)^{2}}{2}+\frac{(\gamma+1)\left(u_{j}^{n}\right)^{3}}{2}\right)\right] \\
& \leqslant-\sum_{j} \Delta x\left(\frac{\left(\Delta_{+}^{x} \rho\left(j \Delta x, t_{n}\right)\right.}{\Delta x}\right)\left(u_{j+1}^{n} \theta_{j} \Delta_{+} f\left(u_{j}^{n}\right)-\lambda_{n}\left(\Delta_{+}\left(1-\theta_{j}\right) f\left(u_{j}^{n}\right)\right)^{2}\right) .
\end{aligned}
$$

We now have analogues of Theorem 3.2 and 3.3.

THEOREM 4.2. The solution to (3.6)-(3.12) with (4.4), (4.6) replacing (3.7) satisfies the $L^{2}$ stability estimates of Theorem 3.1 .

THEOREM 4.3. Suppose $\varphi_{j k}^{n}$ is determined in the same way as in the previous theorem and $\varphi_{j k}^{n}, u_{j k}^{n}$ and $v_{j k}^{n}$ converge boundedly, a.e. as $\Delta x, \Delta y, \Delta t_{n} \rightarrow 0$ to $\Phi$, $u$ and $v$. Then $\Phi$ is weak solution of (3.1) which satisfies the entropy inequality of Theorem 3.2.

5. A One-Dimensional Model Problem. Most of the important phenomena in the small disturbance equation, and its approximations occur in the $x$-direction. The nonlinearity and the switch in the difference formulas depend only on the $x$-derivative. Thus, it is of interest to study the $y$-independent problem.

$$
\Phi_{x t}=\left(K \Phi_{x}-1 / 2(\gamma+1) \Phi_{x}^{2}\right)_{x}
$$

or with $u=\Phi_{x}$

$$
u_{t}=\left(K u-1 / 2(\gamma+1) u^{2}\right)_{x}
$$

where $u$ is a function of $x$ and $t$, and the corresponding difference approximations.

Consider also

$$
u_{t}=-f(u)_{x}
$$

where $f$ is convex and $f^{\prime}(\bar{u})=0$ and

$$
2 w_{t}=-\left(w^{2}\right)_{x}
$$

The equation (5.4) is derived from (5.1) after the transformations

$$
\Phi_{x} \rightarrow w+\frac{K}{\gamma+1}, \quad t \rightarrow(\gamma+1) t .
$$

Normalize the independent variables such that $0 \leqslant x \leqslant 1, t \geqslant 0$. The difference scheme corresponding to (5.3) and the $x$-step in the splitting scheme (3.7) is 


$$
u_{j}^{n+1}=u_{j}^{n}-\lambda_{n} \Delta_{+} f\left(\min \left(u_{j}^{n}, \bar{u}\right)\right)-\lambda_{n} \Delta_{-} f\left(\max \left(u_{j}^{n}, \bar{u}\right)\right),
$$

where $\lambda_{n}=\Delta t_{n} / \Delta x$. The linear stability condition is $\max _{j, n}\left|\lambda_{n} f^{\prime}\left(u_{j}^{m}\right)\right|=\epsilon_{0} \leqslant 1$.

With the boundary condition

$$
u_{0}=u^{L}, \quad u_{N}=u^{R}
$$

for $f\left(u^{L}\right)=f\left(u^{R}\right)$ we can show that there exists a unique steady state solution for (5.5).

THEOREM 5.1. A solution of (5.5) satisfying the boundary conditions is a steady solution, if and only if it is of the form

$$
u_{j} \equiv u^{L}, \quad 0 \leqslant j \leqslant j_{0}
$$

$$
u_{j_{0}+1}=a
$$

$$
u_{j_{0}+2}=b
$$

$$
u_{j} \equiv u^{R}, \quad j \geqslant j_{0}+3 \text { for some } j_{0},
$$

where $a$ and $b$ satisfy

$$
u^{R} \leqslant b \leqslant \bar{u} \leqslant a \leqslant u^{L}
$$

and

$$
f\left(u^{L}\right)+f(\bar{u})=f\left(u^{R}\right)+f(\bar{u})=f(a)+f(b) .
$$

The solution is made unique by requiring that

$$
\sum_{j=1}^{N} u_{j} \Delta x=A
$$

for $u^{R} \leqslant A \leqslant u^{L}$

Proof. The function $u_{j}^{n} \equiv u_{j}$ is a steady solution of (5.5) if and only if

$$
\int_{u_{j}}^{u_{j+1}} f^{\prime}(s) \chi(s) d s=\int_{u_{j-1}}^{u_{j}} f^{\prime}(s)(\chi(s)-1) d s, \quad j=1, \ldots, N-1,
$$

with

$$
u_{0}=u^{L}, \quad u_{N}=u^{R}, \quad u^{L}>\bar{u}>u^{R}, \quad f\left(u^{L}\right)=f\left(u^{R}\right)
$$

(Recall $\chi(s)$ was defined in Lemma 2.1.) We can construct such solutions as follows. Let $u_{j} \equiv u^{L}, j \leqslant j_{0}$, with $0<j_{0} \leqslant N-1$. Then Eq. (5.10) is valid for $j \leqslant$ $j_{0}-2$, while for $j=\ddot{j}_{0}$ we have

$$
\int_{u}^{u_{j_{0}+1}} \chi(s) f^{\prime}(s) d s=0
$$

This is valid if and only if $u_{j_{0}+1} \leqslant \bar{u}$. For $j=j_{0}+1$ we have 


$$
\begin{aligned}
\int_{u_{j_{0}+1}}^{u_{j_{0}+2}} f^{\prime}(s) \chi(s) d s & =\int_{u^{L}}^{u_{j_{0}+1}} f^{\prime}(s)(\chi(s)-1) d s \\
& =f\left(u^{L}\right)-f\left(u_{j_{0}+1}\right) \neq 0 .
\end{aligned}
$$

Thus, $u_{j_{0}+2}=\bar{u}$ and we have

$$
f\left(u_{j_{0}+2}\right)-f(\bar{u})=f\left(u^{L}\right)-f\left(u_{j_{0}+1}\right) .
$$

For $j=j_{0}+2$ we have

$$
\begin{aligned}
\int_{u_{j_{0}+2}}^{u_{j_{0}+3}} f^{\prime}(s) \chi(s) d s & =\int_{u_{j_{0}+1}}^{u_{j_{0}+2}} f^{\prime}(s)(\chi(s)-1) d s \\
& =f\left(u_{j_{0}+1}\right)-f(\bar{u}) \\
& =f\left(u^{L}\right)-f\left(u_{j_{0}+2}\right) \quad(\text { by }(5.13)) \\
& =f\left(u^{R}\right)-f\left(u_{j_{0}+2}\right) .
\end{aligned}
$$

Thus, one solution of (5.14) is $u_{j_{0}+3}=u^{R}$. By the geometry of $f(u)$ any other solution would have to be $u_{j_{0}+3}>\bar{u}$ so we would have

$$
f(\bar{u})-f\left(u_{j_{0}+2}\right)=f\left(u^{R}\right)-f\left(u_{j_{0}+2}\right) .
$$

But $f\left(u^{R}\right)>f(\bar{u})$, and hence $u_{j_{0}+3}=u_{R}$.

It is easy to see that if two consecutive points on a steady solution of (5.5) satisfy $u_{j_{1}} \leqslant \bar{u}, u_{j_{1}+1} \leqslant \bar{u}$, then $u_{j} \equiv u_{j_{1}+1}$ for $j \geqslant j_{1}+1$;

Thus, it follows that all steady solutions of this type are as described in (5.7), (5.8).

It remains to construct solutions for $j_{0}=0$. We then have

$$
\begin{aligned}
\int_{u_{1}}^{u_{2}} f^{\prime}(s) \chi(s) d s & =\int_{u_{0}}^{u_{1}} f^{\prime}(s)(\chi(s)-1) d s \\
= & \begin{cases}f\left(u^{L}\right)-f\left(u_{1}\right) & \text { if } u_{1} \geqslant \bar{u} \\
f\left(u^{L}\right)-f(\bar{u}) & \text { if } u_{1}<\bar{u}\end{cases}
\end{aligned}
$$

In either case, the right side is $\neq 0$ which means, if $u_{1} \geqslant \bar{u}$, that $u_{2} \leqslant \bar{u}$; and we now have

$$
f\left(u_{2}\right)-f(\bar{u})=f\left(u^{L}\right)-f\left(u_{1}\right)
$$

Then, as in the previous case for which $0<j_{0}<N-1$ we must have

$$
u_{0}=u^{L}, \quad u_{1}=a, \quad u_{2}=b, \quad u_{3}=u^{R}, \text { etc. }
$$

The last possibility is $u_{1}<\bar{u}$, and we must have 


$$
\int_{u_{1}}^{u_{2}} f^{\prime}(s) \chi(s) d s=f\left(u^{L}\right)-f(\bar{u})>0 .
$$

This means that $u_{2}<u_{1}$, and they are chosen so that

$$
f\left(u_{2}\right)-f\left(u_{1}\right)=f\left(u^{L}\right)-f(\bar{u}) .
$$

The next equation tells us that $u_{3}=u_{2}=u^{R}$, which means that $u_{1}=\bar{u}$, which is a contradiction.

Next we wish to choose $j_{0}, a$, and $b$ so that the sum takes on a fixed value

$$
u^{R} \leqslant \sum_{j=1}^{N} u_{j} \Delta x=A \leqslant u^{L} .
$$

Define $x_{0}$ to be the value of $x$ at which the limit function is discontinuous

$$
u^{L} x_{0}+u^{R}\left(1-x_{0}\right)=A, \quad\left(u^{L}-u^{R}\right) x_{0}=A-u^{R},
$$

so

$$
x_{0}=\left(A-u^{R}\right) /\left(u^{L}-u^{R}\right)
$$

We wish to find $j_{0}, a$, and $b$ so that

$$
\begin{gathered}
u^{L} j_{0} \Delta x+(a+b) \Delta x+u^{R}\left(N-j_{0}-2\right) \Delta x \\
=A=u^{L} x_{0}+u^{R}\left(1-x_{0}\right) .
\end{gathered}
$$

When $(a+b)$ takes on its maximum (minimum) possible value subject to (5.33), we choose $j_{0}$ so that the left side of (5.21) is greater than (less than) or equal to the right side of (5.22)

$$
\begin{gathered}
(a+b)_{\max }=u^{L}+\bar{u}, \quad(a+b)_{\min }=u^{R}+\bar{u} \\
u^{L}\left(j_{0}+1\right)+\bar{u}+u^{R}\left(N-j_{0}-2\right) \geqslant u^{L} \frac{x_{0}}{\Delta x}+u^{R}\left(N-\frac{x_{0}}{\Delta x}\right) \\
\geqslant u^{L} j_{0}+\bar{u}+u^{R}\left(N-j_{0}-1\right),
\end{gathered}
$$

or

$$
\left(j_{0}+1\right)+\frac{\bar{u}-u^{R}}{u^{L}-u^{R}} \geqslant \frac{x_{0}}{\Delta x} \geqslant j_{0}+\frac{\bar{u}-u^{R}}{u^{L}-u^{R}} .
$$

Thus, we can take $j_{0}$ to be the smallest integer such that

$$
j_{0}>\frac{x_{0}}{\Delta x}-1-\frac{\bar{u}-u^{R}}{u^{L}-u^{R}} .
$$

Let us go back to the time-dependent case with the scheme (5.5) approximating (5.3). In Theorem 5.2 we present some properties of the scheme.

THEOREM 5.2. The scheme (5.5) for $f$ convex is first order accurate, monotone, and in conservation form. Moreover, if we write 


$$
u_{j}^{n+1}=G\left(u_{j+1}^{n}, u_{j}^{n}, u_{j}^{n}\right)=u_{j}^{n}-\lambda_{n} h\left(u_{j+1}^{n}, u_{j}^{n}\right)+\lambda_{n} h\left(u_{j}^{n}, u_{j-1}^{n}\right),
$$

then $G$ is a $C^{1}$ and piecewise smooth function of its arguments.

Proof. We again define

$$
\begin{array}{ll}
\chi(u) \equiv 0 & \text { if } f^{\prime}(u) \geqslant 0 \\
\chi(u) \equiv 1 & \text { if } f^{\prime}(u)<0 .
\end{array}
$$

Then (5.25) may be rewritten

$$
\begin{aligned}
u_{j}^{n+1} & =u_{j}^{n}-\lambda_{n} \int_{u_{j}^{n}}^{u_{j+1}^{n}} f^{\prime}(s) \chi(s) d s-\lambda_{n} \int_{u_{j-1}^{n}}^{u_{j}^{n}} f^{\prime}(s)(1-\chi(s)) d s \\
& \equiv G\left(u_{j+1}^{n}, u_{j}^{n}, u_{j-1}^{n}\right)
\end{aligned}
$$

with

$$
\begin{aligned}
\frac{\partial G}{\partial u_{j+1}} & =-\lambda f^{\prime}\left(u_{j+1}\right) \chi\left(u_{j+1}\right), \\
\frac{\partial G}{\partial u_{j}} & =1+\lambda f^{\prime}\left(u_{j}\right)\left(2 \chi\left(u_{j}\right)-1\right), \\
\frac{\partial G}{\partial u_{j-1}} & =\lambda f^{\prime}\left(u_{j-1}\right)\left(1-\chi\left(u_{j-1}\right)\right) .
\end{aligned}
$$

Thus the scheme is monotone, and $G$ is $C^{1}$ and piecewise smooth.

Letting $f^{\prime}(s) \chi(s)=f^{\prime}(s)+f^{\prime}(s)(\chi(s)-1)$ in (5.27) gives us

$$
u_{j}^{n+1}=u_{j}^{n}-\lambda_{n} \Delta_{+} f\left(u_{j}^{n}\right)+\lambda_{n} \Delta_{-} \int_{u_{j}^{n}}^{u_{j+1}^{n}} f^{\prime}(s)(1-\chi(s)) d s .
$$

We may take

$$
h\left(u_{j+1}, u_{j}\right)=f\left(u_{j+1}\right)-\int_{u_{j}}^{u_{j+1}} f^{\prime}(s)(1-\chi(s)) d s,
$$

and conservation form is immediate.

Let

$$
g(u)=\int_{\bar{u}}^{u} f^{\prime}(s)(1-\chi(s)) d s .
$$

We may rewrite (5.29) as

$$
u_{j}^{n+1}=u_{j}^{n}-\lambda_{n} \Delta_{+} f\left(u_{j}^{n}\right)+\lambda_{n} \Delta_{-} \Delta_{+} g\left(u_{j}^{n}\right) .
$$

Since $g^{\prime}(x)$ is Lipschitz continuous, it follows that if $u$ is Lipschitz continuous in a neighborhood of $u_{j}^{n}$ then

$$
\Delta_{+} \Delta_{-} g\left(u_{j}^{n}\right)=O\left(h^{2}\right)
$$

First order accuracy is now immediate. 
Important consequences of monotonicity are:

(A) Monotone schemes form an $L^{1}$ contraction [5], i.e., if

$$
u_{j}^{n+1}=G\left(u_{j+k}^{n}, \ldots, u_{j-k}^{n}\right), \quad v_{j}^{n+1}=G\left(v_{j+k}^{n}, \ldots, v_{j-k}^{n}\right),
$$

either on an infinite region $-\infty<j<\infty$, or a compact region with periodic boundary conditions, then

$$
\sum_{j}\left|u_{j}^{n+1}-v_{j}^{n+1}\right| \Delta x \leqslant \sum_{j}|| u_{j}^{n}-v_{j}^{n} \mid \Delta x
$$

This estimate has been used [3] to prove:

(B) Solutions to monotone difference schemes converge as $\max \left(\Delta t_{n}, \Delta x\right) \rightarrow$ 0 to weak solutions of (5.3).

(C) Bounded a.e. limits as $\max \left(\Delta t_{n}, \Delta x\right) \rightarrow 0$ of solutions of (5.5) satisfy the entropy inequality

$$
\frac{\partial}{\partial t} \frac{u^{2}}{2}+\frac{\partial}{\partial x} F(u) \leqslant 0
$$

Here

$$
F(u)=\frac{-K u^{2}}{2}+\frac{(\gamma+1) u^{3}}{3} \text { for } f(u)=-K u+(\gamma+1) \frac{u^{2}}{2} .
$$

(D) Solutions of (5.5) satisfy the ordering principle:

$$
\text { if } u_{j}^{n} \leqslant v_{j}^{n}, \text { then } u_{j}^{n+1} \leqslant v_{j}^{n+1} .
$$

We may use property (D) to show

Proposition 5.1. If $u_{j}^{n}$ satisfies (5.5) for $j=1, \ldots, N-1$, with boundary conditions $u_{0}^{n} \equiv u^{L}, u_{N}^{n} \equiv u^{R}, n \geqslant 0$ for $f\left(u^{L}\right)=f\left(u^{R}\right), u^{L}>u^{R}$, and initial conditions $u^{R} \leqslant u_{j}^{0} \leqslant u^{L}$, then $u_{j}^{n}$ satisfies (5.5) on the infinite interval $-\infty<j<\infty$ with

$$
\begin{gathered}
u_{j}^{n} \equiv u^{L}, \quad j \leqslant 0, \\
u_{j}^{n} \equiv u^{R}, \quad j \geqslant N .
\end{gathered}
$$

Using a modified version of the proofs in [7], James Ralston has shown for our scheme

THEOREM (5.3) (RALSTON). Let $u_{j}^{n}$ solve (5.5) for $-\infty<j<\infty$ with $\lim _{j \rightarrow-\infty} u_{j}$ $=u^{L}, \lim _{j \rightarrow \infty} u_{j}=u^{R}$ and

$$
\sum_{j=-\infty}^{0}\left|u_{j}-u^{L}\right|<\infty, \quad \sum_{j=1}^{\infty}\left|u_{j}-u^{R}\right|<\infty .
$$

Then there exists a steady solution $u_{j}^{\infty}$ of the type (5.7) with

$$
\lim _{n \rightarrow \infty} u_{j}^{n}=u_{j}^{\infty}
$$

COROLlARY (5.1). Solutions of the discrete initial boundary value problem converge as $n \rightarrow \infty$ to the unique steady solution $u_{j}^{\infty}$ which has the property 


$$
\sum_{j=0}^{N} u_{j}^{\infty}=\sum_{j=0}^{N} u_{j}^{0}
$$

We have calculated approximate solutions of the model problem (5.4) using several different schemes. For one set of experiments the initial values were chosen to contain an entropy violating shock

$$
w(x, 0)=\left\{\begin{array}{cl}
1, & 0 \leqslant x \leqslant 1 / 4,1 / 2<x<3 / 4, \\
-1, & 1 / 4<x \leqslant 1 / 2,3 / 4<x \leqslant 1 .
\end{array}\right.
$$

We compared the following difference schemes:

(a) Engquist-Osher Scheme (5.5).

(b) Entropy condition satisfying Cole-Murman scheme (2.11), (4.4).

(c) Cole-Murman scheme (2.11).

(d) Lax-Friedrichs scheme

$$
w_{j}^{n+1}=\frac{1}{2}\left(w_{j+1}^{n}+w_{j-1}^{n}\right)-\frac{\lambda}{2} D_{0}^{x}\left(w_{j}^{n}\right)^{2} .
$$

(e) Lax-Wendroff scheme with nonlinear dissipation; see [10]. The following numerical boundary conditions were used;

$$
w_{0}^{n}=1, \quad w_{N}^{n}=-\sum_{j=0}^{N-1} w_{j}^{n} .
$$

With increasing $n$ the solution of (a), (b) and (e) converged to a steady state, which essentially agreed with the analytic solution $w(x)$ for $t$ large enough. See Table 5.1 for details

$$
w(x)=\left\{\begin{array}{cc}
1, & 0 \leqslant x<1 / 2 \\
-1, & 1 / 2<x<1 .
\end{array}\right.
$$

The Cole-Murman scheme (c) has the entropy violating initial values (5.38) as steady state solutions and the Lax-Friedrichs scheme (d) oscillates between two states in the limit $n \rightarrow \infty$. One of the states is given in Table 5.1.

TABLE 5.1

The discrete shock profiles, as functions of $x_{\boldsymbol{j}}$, for the analytic solution and the approximation (a), (b), (d) and (e) as $n \rightarrow \infty$. $\Delta t / \Delta x=0.5, \Delta x=1 / 21$.
(a) $\begin{array}{llll}1.00 & 1.00 & 1.00 & 1.00 \\ 1.00 & 1.00 & 1.00 & 1.00\end{array}$
\begin{tabular}{l|rrrrr}
1.00 & -1.00 & -1.00 & -1.00 & -1.00 & -1.00 \\
0.71 & -0.71 & -1.00 & -1.00 & -1.00 & -1.00
\end{tabular}
(b) $1.00 \quad 1.00 \quad 1.00 \quad 1.00$
$0.78-0.78 \quad-1.00 \quad-1.00 \quad-1.00 \quad-1.00$
(d) 0.92
$0.99 \quad 0.48$
0.90
$-0.42 \quad 0.42$
1.05
$0.87 \mid-0.87$
$\begin{array}{llll}-0.90 & -0.48 & -0.99 & -0.92\end{array}$
(e) 1.00
$1.01 \quad 0.98$

1.05


Note here that the nonswitching schemes Lax-Wendroff and Lax-Friedrichs spread the shock over a larger domain. Furthermore, the shock profile for those schemes is no longer monotone.

Remark 1. The qualitative information in the table is valid for other values of $\Delta t$ and $\Delta x$. It is also valid if the initial data is perturbed. For example, if random noise of amplitude 0.1 is added to the initial values the solutions of (a), (b) and (e) still converge to the profiles in the table. The scheme (c), will converge to a steady state close to (5.38) containing an entropy violating shock. However, if the initial values are drastically changed, for example to

$$
w(x, 0)=1-2 x
$$

even the Cole-Murman scheme will converge to the right solution with the error only spread over at most two grid points in space.

The rate of convergence is given in Table 5.2.

TABLE 5.2

The $l_{1}$-norm of the error $w_{j}^{n}-w\left(x_{j}\right),(w(x)$ is the steady state solution)

$$
\Delta t / \Delta x=0.5, \Delta x=1 / 21 \text {. }
$$

$n=\quad 20 \quad 40 \quad 60 \quad 80$

$\begin{array}{lllll}\text { (a) } & 0.500 & 0.036 & 0.029 & 0.029 \\ \text { (b) } & 0.460 & 0.025 & 0.023 & 0.023 \\ \text { (d) } & 0.365 & 0.225 & 0.215 & 0.215 \\ \text { (e) } & 0.667 & 0.189 & 0.024 & 0.023\end{array}$

The scheme (b) gives a better result than scheme (a) in this case. In general, this might be somewhat misleading because it is possible to take longer time steps with (a). The computational stability limits for (a), (c), (d) and (e) are $\Delta t / \Delta x \leqslant 1$, but for (b) it is $\Delta t / \Delta x \leqslant 0.75$.

Remark 2. In [7] Jennings showed that a strictly monotone scheme converges to a steady state as $n \rightarrow \infty$. The Lax-Friedrichs scheme (d) is monotone but oscillates in the limit $n \rightarrow \infty$. There is no contradiction since (d) is not strictly monotone. If the scheme (5.39) is written in the form

$$
w_{j}^{n+1}=G\left(w_{j+1}^{n}, w_{j}^{n}, w_{j-1}^{n}\right),
$$

we have $\partial G / \partial w_{j}^{n}=0$, not $>0$. The scheme does not couple grid points where $n+j$ is odd with points where $n+j$ is even. As pointed out by James Ralston, regarded as an algorithm of the form

$$
u_{j}^{n+2}=G\left(u_{j+2}^{u}, u_{j}^{n}, u_{j-2}^{u}\right),
$$

the Lax-Friedrichs scheme is strictly monotone and the convergence results of [7] are valid. 
6. A Numerical Example and Some Computational Comments. We have applied the ADI-scheme of Section 3 with the original Cole-Murman switch (2.11), and the modified switch (2.8) to the simple test problem of transonic flow around a symmetric airfoil. The computational domain $0 \leqslant x \leqslant 5 c, 0 \leqslant y \leqslant 2 c$, where $c$ is the chord, is covered by a regular grid. The airfoil is located at $2 c \leqslant x \leqslant 3 c, y=0$. The initial values and boundary conditions are

$$
\begin{aligned}
& \Phi(x, y, 0)=0, \quad 0 \leqslant x \leqslant 5 c, 0 \leqslant y \leqslant 2 c, \\
& \Phi(0, y, t)=0, \quad 0 \leqslant y \leqslant 2 c, t>0, \\
& \Phi_{x}(5 c, y, t)=0, \quad 0 \leqslant y \leqslant 2 c, t>0, \\
& \Phi_{y}(x, 2 c, t)=0, \quad 0 \leqslant x \leqslant 5 c, t>0, \\
& \Phi_{y}(x, 0, t)= \begin{cases}0, & 0 \leqslant x \leqslant 2 c, 3 c \leqslant x \leqslant 5 c, t>0, \\
h(x), & 2 c \leqslant x \leqslant 3 c, \quad t>0\end{cases}
\end{aligned}
$$

The shape of the airfoil is given by the function $h(x)$, and it is chosen as part of a sine function. The $y$-derivative of $\Phi$ is discontinuous at $y=0, x=2 c, 3 c$. The derivatives in the boundary conditions were replaced by one-sided differences. Several computations were performed with both types of switches. Different far field Mach numbers and different grid sizes were used. The calculations were continued until steady state was reached. (No change in the 4th decimals of $\varphi$ during 10 consecutive iterations.)

The main conclusion from these calculations is that both types of switches give approximations to $\Phi$ which are very close to each other. When the initial values are such that the Cole-Murman scheme does not produce an expansion shock the convergence characteristics for the solutions corresponding to both switches are similar. For both methods the shock profile was sharp as is well known for approximations using the ColeMurman scheme. With the modified switch slightly longer timesteps could be used without causing instabilities.

In Table 6.1 we give the velocity $\Phi_{x}$ at the airfoil as a function of $x$. The result is typical for a coarse grid calculation. We used a $60 \times 24$ grid in this example, and $\varphi_{x}$ is presented for timestep 200 where the solution is close to the steady state. The Mach number was 0.85 and the CFL number was 0.7 .

TABLE 6.1

The velocity $D_{+}^{x} \varphi_{j 0}^{200}$ as a function of $x_{j}, 2 c \leqslant x_{j} \leqslant 3 c$.

$\mathrm{C}-\mathrm{M}$ : The scheme (2.11), (3.3)-(3.11), E-0: The scheme (3.7)-(3.11)

\begin{tabular}{|r|rrrrrrr|}
\hline C-M & -0.47 & 0.09 & 0.69 & 1.45 & 1.86 & 2.32 & 2.72 \\
\cline { 2 - 8 } & 3.06 & 3.21 & 3.43 & 3.16 & -0.57 & -1.23 & \\
\hline E-O & -0.47 & 0.10 & 0.69 & 1.45 & 1.85 & 2.31 & 2.72 \\
\cline { 2 - 8 } & 3.06 & 3.20 & 3.43 & 3.01 & -0.48 & -1.23 & \\
\hline
\end{tabular}


Computational Comments. The algorithms which we have analyzed in this paper can, and of course should, be modified in applications to suit different engineering needs. For example, the limitation on the timestep is naturally costly if the scheme is used only for steady state calculations. What follows is some remarks on how the new entropy guaranteeing switch can be used where the theory does not apply directly.

1. The computational grid does not need to be uniform. A nonuniform grid with smaller step sizes close to the airfoil and especially close to the ends of the airfoil can increase the accuracy (see [1], [8]). Most of the difference formulas are still valid after minor modifications, for example,

$$
D_{+}^{y} D_{-}^{y} \varphi_{k} \rightarrow \frac{2}{\Delta y_{k}+\Delta y_{k+1}}\left(\frac{\varphi_{k+1}-\varphi_{k}}{\Delta y_{k}}-\frac{\varphi_{k}-\varphi_{k-1}}{\Delta y_{k-1}}\right) \Delta y_{k}=y_{k+1}-y_{k} .
$$

2. In order to speed up convergence when steady state solutions are being calculated, the longer timesteps of implicit schemes are needed. It is easy to change the $x$-differencing to be semi-implicit; see [1]. The new switch can of course still be used. It is furthermore not necessary to have a fixed $\Delta t$. The timestep may vary between the iterations and also between different grid points. In this case the algorithm need not be consistent with the time-dependent problem.

3. For time-dependent problems artificial reflections from the outer computational boundaries can cause a lot of trouble. These reflections can be reduced by using radiating boundary conditions; see [4]. For example, $\varphi_{y}=0$ at the outer boundary $y=2 c$ in our test problem can be replaced by $\varphi_{y}+a \varphi_{x}=0$, where $a$ is a constant or a function of $\varphi_{x}$.

4. There are several ways of changing the algorithm in order to obtain second order accuracy. For the $y$-sweep the following approximation is second order and stable as a separate Cauchy problem

$$
D_{-}^{x}\left(\varphi_{j, k}^{n+1}-\varphi_{j, k}^{n}\right)=\frac{\Delta t}{4} D_{+}^{y} D_{-}^{y}\left(\varphi_{j, k}^{n+1}+\varphi_{j-1, k}^{n+1}+\varphi_{j, k}^{n}+\varphi_{j-1, k}^{n}\right) .
$$

The $x$-sweep can easily be changed to be second order; see [1]. It is also possible to use a standard method such as Lax-Wendroff for the $x$-differencing.

We shall present a rigorous analysis of certain second order methods in a succeeding paper.

Department of Mathematics

University of California, Los Angeles

Los Angeles, California 90024

1. W. F. BALlHAUS \& P. M. GOORJIAN, Implicit Finite Difference Computation of Unsteady Transonic Flow About Airfoils Including the Treatment of Irregular Shock Wave Motions, A.I.A.A. paper No. 77-205, 1977.

2. J. D. COLE, "Modern developments in transonic flow," SIAM J. Appl. Math., v. 29, 1975, pp. 763-786.

3. M. G. CRANDALL \& A. MAJDA, "Monotone difference approximations for scalar conservation laws." (To appear.)

4. B. ENGQUIST \& A. MAJDA, "Numerical radiation boundary conditions for unsteady transonic flow," J. Computational Phys. (Submitted.) 
5. A. HARTEN, J. M. HYMAN \& P. D. LAX, "On finite difference approximations and entropy conditions for shocks," Comm. Pure Appl. Math., v. 29, 1976, pp. 297-322.

6. A. JAMESON, "Numerical solutions of nonlinear partial differential equations of mixed type," Numerical Solutions of Partial Differential Equations III, Academic Press, New York, 1976, pp. $275-320$.

7. G. JENNINGS, “Discrete shocks,” Comm. Pure Appl. Math., v. 27, 1974, pp. 25-37.

8. J. A. KRUPP \& J. D. COLE, Studies in Transonic Flow IV, Unsteady Transonic Flow, UCLA Eng. Dept. Rep. No. 76/04, 1976.

9. P. D. LAX \& B. WENDROFF, "Systems of conservation laws," Comm. Pure Appl. Math., v. 23, 1960, pp. 217-237.

10. A. MAJDA \& S. OSHER, "Numerical viscosity and the entropy condition," Comm. Pure Appl. Math. (Submitted.)

11. M. S. MOCK, "Systems of conservation laws of mixed type," J. Differential Equations. (Submitted.)

12. E. M. MURMAN \& J. D. COLE, "Calculations of plane steady transonic flows," AIAA J., v. 9, 1971 , pp. 114-121. 